\title{
ARTÍCULO
}

\section{MOVILIDAD INTRAGENERACIONAL DEL INGRESO EN CHILE*}

\author{
Claudio Sapelli \\ P. Universidad Católica de Chile
}

\begin{abstract}
Resumen: En este trabajo se estiman los diferentes índices de movilidad intrageneracional para Chile sobre la base del panel Casen. A la luz de los resultados se evalúan las conclusiones de la literatura que se basan principalmente en la discusión de la matriz de transición. Se concluye que la lectura de los datos en una parte de la literatura es equivocada. Se hace una simulación que permite demostrar que la matriz de transición chilena podría ser consecuencia de un proceso de shocks aleatorios sobre la distribución del ingreso efectiva, demostrando que las características diferenciales de ella son consecuencia de la forma de la distribución del ingreso. Del análisis que se hace de las matrices de transición se concluye que en Chile hay alta movilidad y que no hay evidencia de barreras a la misma entre diferentes grupos de ingreso.
\end{abstract}

Palabras clave: movilidad, desigualdad.

Clasificación JEL: J3, D6.

Recibido: junio 2013; aceptado: agosto 2013.

Claudio Sapelli. Ph. D. en Economía, Universidad de Chicago. Profesor del Instituto de Economía de la P. Universidad Católica de Chile.

* Agradezco la importante colaboración de Gastón Illanes en el desarrollo de este trabajo. A su vez agradezco los comentarios de dos referees de Estudios Públicos y de mis colegas del Instituto de Economía de la PUC. Finalmente, agradezco la ayuda de Fernanda Rojas. 


\title{
INTRA-GENERATIONAL MOBILITY OF INCOME IN CHILE
}

\begin{abstract}
This paper estimates the different intragenerational mobility indicators for Chile on the basis of the Casen panel. Conclusions from the literature are evaluated in light of results that are based mainly on a discussion of the transition matrix. It is concluded that there is a mistaken interpretation of the data in part of the literature. A simulation is made that demonstrates that the Chilean transition matrix could be the consequence of random shocks to the effective distribution of income, demonstrating that the differences are the result of how income is distributed. It is concluded from an analysis of the transition matrixes that there is high mobility in Chile and no evidence of barriers to that mobility among different income groups.
\end{abstract}

Keywords: mobility, inequality.

JEL Classification: J3, D6.

Received: June 2013; accepted: August 2013.

$\mathrm{L}$

a desigualdad del ingreso es un tema que ha sido estudiado numerosas veces para Chile, especialmente desde una visión estática, donde se evalúa la distribución del ingreso en un momento dado. Este trabajo busca complementar dichos trabajos aportando una visión dinámica sobre la distribución del ingreso, estudiando cómo los individuos se mueven a lo largo de ésta en el tiempo. Con este objetivo, este trabajo estudia la movilidad intrageneracional del ingreso en Chile, lo que consiste en seguir a individuos a lo largo del tiempo y observar cómo ellos se mueven a lo largo de la distribución del ingreso.

La primera interrogante que surge al enfrentarse a este tema es: ¿qué es lo deseable? Es decir, ¿es preferible una sociedad con alta movilidad del ingreso, lo que implica que muchos individuos avanzan en la distribución del ingreso pero también que otros retroceden, o es preferible una sociedad un poco más rígida, donde el ranking de ingreso de las personas se mueve más lentamente o se mantiene relativamente constante?

Este trabajo sigue a la literatura internacional (Fields y Ok, 1996 y 2001, y Gottschalk y Spolaore, 2002) en postular que la evaluación del nivel de movilidad intrageneracional del ingreso de un país depende crucialmente de una definición normativa del "mejor escenario". Sin embargo, como se verá, si bien hay discrepancia respecto a qué 
constituye una movilidad "deseable", la rigidez nunca es considerada "buena". La discusión en la literatura radica en cuál es la naturaleza de la movilidad que se considera deseable.

La interpretación normativa del nivel de movilidad del ingreso de un país dependerá de si se estima que una sociedad perfectamente móvil es aquella donde el ingreso futuro no está determinado por el ingreso presente (conocido como temporal independence, independencia intertemporal), o si es aquella donde la distribución del ingreso se revierte, es decir, aquella donde los más ricos se transforman en menos ricos (caen en el ranking) y viceversa (conocido como reversal), o si es una combinación de ambas. Más que postular una respuesta "correcta" a la disyuntiva anterior, este trabajo analiza las implicancias de las dos visiones previamente mencionadas y los alcances de éstas para la interpretación de los resultados para Chile.

En este contexto, nos interesa discutir la posición de Contreras, Cooper, Herman y Neilson (2004), quienes plantean que los altos niveles de movilidad intrageneracional del ingreso encontrados para Chile implican mayor vulnerabilidad, algo indeseable. Sin embargo, veremos que los resultados para Chile muestran altos niveles de independencia intertemporal, y que los movimientos que podrían ser interpretados como vulnerabilidad (individuos cuyo ingreso sube y luego baja, o viceversa), también podrían ser interpretados como reversal. En la literatura la discusión radica en cuál de estos procesos es deseable, pero no en que ninguno lo sea. Afirmar que la alta movilidad no es deseable, independientemente de la forma que ella tome, como al parecer hacen Contreras et al., genera una contradicción al interpretar la movilidad como vulnerabilidad (algo indeseable), ya que si se considera que lo importante es la independencia intertemporal, Chile muestra altos niveles de ella, pero si se piensa que lo importante es la presencia de reversal, vulnerabilidad es precisamente lo que se espera encontrar. La sección 1 presentará este argumento en más detalle.

Adicionalmente, a partir de estimadores de movilidad intrageneracional del ingreso, basados en matrices de transición, obtenidos para nuestro país, se ha conjeturado que existe un proceso distinto para los cambios en los ingresos de los individuos del décimo decil (nuevamente, un ejemplo son Contreras, Cooper, Herman y Neilson. 2004). Esto, debido a que se observa que los individuos en el décimo decil tienen una menor probabilidad de transitar hacia otros deciles que los 
individuos de los deciles restantes. Sin embargo, al estimar matrices de transición, este resultado es esperable, pues los individuos en los deciles extremos (primero y décimo) tienen sólo una vía de salida hacia otros deciles: para arriba y para abajo, respectivamente. Luego, una caída fuerte del ingreso no saca a individuos del primer decil, a diferencia de los demás deciles, y del mismo modo un aumento fuerte del ingreso no saca a individuos del décimo decil. Por ende, una mayor probabilidad de permanecer en el decil es precisamente lo que se espera para aquellos individuos que se encuentran en estos deciles. Por otro lado, las particularidades de la distribución del ingreso de Chile hacen pensar que su larga cola derecha puede hacer aún más alta la permanencia en ese decil sin necesitar la hipótesis de barreras. Luego, la pregunta relevante es si, condicional en la distribución del ingreso de Chile, la probabilidad de mantenerse en el décimo decil es lo suficientemente alta como para ser necesariamente generada por un proceso aleatorio distinto o no. Esta cuestión será explorada en la sección 2.

Un tema relacionado con el de la movilidad intrageneracional del ingreso es el de la movilidad intergeneracional del mismo. La movilidad intrageneracional se refiere a la movilidad de una población dada, mientras que la movilidad intergeneracional se refiere al grado en el que los ingresos de los hijos están determinados por los de sus padres. La deseabilidad de la segunda es clara: mayor movilidad intergeneracional es "buena", pues implica que los ingresos de los hijos pueden estar determinados por su esfuerzo individual en lugar de estar determinados por los ingresos de sus padres. Mayor movilidad intergeneracional se interpreta como mayor igualdad de oportunidades. Núñez y Risco (2004) estiman un indicador de la movilidad intergeneracional del ingreso para Chile, y utilizando comparaciones internacionales encuentran que ésta es baja. En este escenario, la interpretación de la movilidad intrageneracional del ingreso como vulnerabilidad reaparece, pues la combinación de ésta con el bajo nivel de movilidad intergeneracional del ingreso encontrado por Núñez y Risco (2004) lleva a la conclusión de que Chile se encuentra en el "peor de los mundos", un escenario con alta rigidez intergeneracional y alta vulnerabilidad a lo largo del ciclo de vida. Sin embargo, este escenario es cuestionable por dos motivos.

Primero, la noción de movilidad como vulnerabilidad es cuestionable, como veremos en la sección 1; y segundo, la estimación de la movilidad intergeneracional del ingreso adolece de sesgos importantes. 
La sección 3 explora estos temas. En particular, se sostiene que la movilidad intergeneracional de la educación podría ser un mejor estimador del nivel de rigidez intergeneracional de una sociedad, movilidad que, según Sapelli (2008), se encuentra en un nivel intermedio a nivel internacional.

En tanto la sección 4 aborda la pregunta de qué está detrás de la movilidad intrageneracional del ingreso observada para Chile, desde el estudio de los cambios en los ingresos de los individuos encuestados en las tres aplicaciones de la Encuesta Panel Casen. Estos cambios son los que generan los indicadores de movilidad antes mencionados, por lo que estudiar sus características y sus determinantes es interesante al momento de evaluar los niveles observados de movilidad intrageneracional. La evidencia provista ahí puede usarse para la discusión de si lo que muestra Chile es reversal o independencia intertemporal, y de si aceptamos una de ellas, o ambas, como una fuente de movilidad evaluada como deseable. Finalmente, la sección 5 concluye.

\section{Movilidad intrageneracional del ingreso}

Existen numerosos estudios, todos basados en la Encuesta Panel Casen, que buscan estimar la movilidad intrageneracional del ingreso para nuestro país. Contreras, Cooper, Herman y Neilson (2004) la estiman mediante matrices de transición para deciles de la distribución del ingreso, utilizando como muestra las Encuestas Panel Casen de 1996 y 2001. Sus principales resultados son que hay alta movilidad en los primeros nueve deciles, y baja movilidad desde y hacia el décimo. A partir de estos resultados, concluyen que los primeros nueve deciles presentan alta vulnerabilidad (considerada indeseable), y que el décimo decil presenta alta rigidez (considerada indeseable). Esta conclusión tiene un problema, no le deja claro al lector si existe un hallazgo positivo posible en estas estimaciones: si la alta movilidad es negativa porque implica vulnerabilidad, y la baja movilidad también es negativa porque implica rigidez, ¿qué forma de movilidad sería deseable?

Castro (2008) estudia la movilidad de ingresos utilizando datos de la Encuesta Panel Casen para los años 1996 y 2001, mediante tres enfoques diferentes. Los primeros dos enfoques, estimar índices de rigidez y utilizar matrices de transición de quintiles, muestran evidencia de alta movilidad durante el período. En tanto, el tercer enfoque 
consiste en utilizar un procedimiento que en dos etapas intenta calcular los determinantes de la movilidad del ingreso. En la primera etapa de este procedimiento, el autor corre una regresión del ingreso por hora en función del género, ubicación, industria donde trabaja, edad, edad al cuadrado, y educación del individuo, y obtiene los valores predichos por esta regresión. Esta regresión tiene como objetivo eliminar el error de medición en la variable de ingreso, pero al predecir sólo el $42 \%$ de su varianza implica que la varianza de los ingresos utilizados en la segunda etapa será menor que la real. Luego, estima un modelo Probit que tiene como variable dependiente una variable dicotómica que indica si la posición relativa del individuo en la distribución del ingreso subió o bajó entre las dos mediciones, y como variables independientes observables de experiencia en el mercado laboral, cambios en la composición familiar, características familiares y características individuales. Sus resultados muestran que el mayor efecto en la movilidad es generado por cambios en la situación laboral, ya sea pasar de empleado a desempleado o viceversa. Al mismo tiempo, encuentra evidencia de que los individuos con educación superior tienen una mejor posición relativa en la distribución del ingreso, y que esta mejor posición disminuye la probabilidad de sufrir una caída en dicha distribución. Adicionalmente, en ambas observaciones encuentra que el hecho de que el jefe de hogar esté casado incrementa la probabilidad de mejorar la posición relativa en un $10 \%$.

Finalmente, Denis, Prieto y Zubizarreta (2007) utilizan las tres aplicaciones de la Encuesta Panel Casen $(1996,2001,2006)$ para estudiar la movilidad de la pobreza durante este período. Mediante la estimación de transiciones desde y hacia la pobreza, encuentran evidencia de alto dinamismo, con mayores salidas desde este estado en el período 2001-2006 que en el período 1996-2001.

En cuanto a evidencia internacional, Sastre y Ayala (2002) utilizan el Panel Study on Income Dynamics (PSID) para Estados Unidos y el European Community Household Panel (ECHP) para la Unión Europea con el objetivo de estimar matrices de transición de deciles de la distribución del ingreso para el período 1993-1997. Regoli, Quintano y Castellano (2003) utilizan la encuesta SHIW del Banco de Italia para llevar a cabo la misma estimación para dicho país, mientras que Ayala, Navarro y Sastre (2002) repiten el ejercicio con las ocho olas de la ECHP para estimar matrices para España, Alemania, Francia, Italia y el 
Reino Unido. Finalmente, Chen (2006) estudia la movilidad del ingreso para EE.UU., el Reino Unido, Canadá y Alemania.

Este trabajo utiliza matrices de transición de deciles para obtener estimadores de la movilidad intergeneracional del ingreso, utilizando como muestra tres olas de la Encuesta Panel Casen: 1996, 2001 y 2006. Este enfoque consiste en tomar una muestra de individuos y ordenarlos en deciles según ingresos, luego observar sus ingresos en otro momento dado, ordenarlos nuevamente en deciles, y posteriormente calcular la probabilidad muestral de pasar de un decil a otro.

Los datos utilizados para este efecto consisten en el ingreso familiar ${ }^{1}$, obtenido a partir de las tres aplicaciones de la Encuesta Panel Casen, el cual es multiplicado por la raíz cuadrada del número de personas del grupo familiar para pasar a ingresos individuales. Adicionalmente, para las estimaciones se elimina al $1 \%$ con ingresos más bajos y al $1 \%$ con ingresos más altos. Ambos ajustes son usuales en la literatura.

Un punto importante a considerar es el problema de la atrición en la muestra. La atrición consiste en que en cada aplicación posterior a la primera de la encuesta, no es posible encontrar a todas las personas encuestadas originalmente. Esto implica que el panel no es balanceado, y podría presentar problemas para la estimación si la selección de los individuos que no son encuestados no es aleatoria. Existen muchos argumentos para creer que efectivamente la "desaparición" es no aleatoria, fundamentalmente que ésta debería ser mayor para los jóvenes y para personas que están sujetas a cambios importantes en su ingreso. Paredes, Prieto y Zubizarreta (2006) estudian la atrición en el contexto de la Encuesta Panel Casen para los años 1996 y 2001, y encuentran que ésta no sólo es no aleatoria, sino que además sesga las estimaciones de índices de movilidad basadas en matrices de transición hacia encontrar menor movilidad que la real. Es decir, una vez que aplican su metodología para corregir por atrición, sus resultados apuntan hacia una mayor movilidad.

Ayala, Navarro y Sastre (2002) estudian el mismo problema en el contexto del ECHP, y al igual que Paredes, Prieto y Zubizarreta (2006) encuentran evidencia de atrición no aleatoria. Para estudiar si esto genera sesgos importantes en la estimación de indicadores de mo-

${ }^{1}$ Los resultados no varían si se usa ingreso autónomo o ingreso total de la familia. Se reportan los resultados con ingreso autónomo. 
vilidad, estos autores desarrollan una metodología ingeniosa: estiman dos matrices de transición para las primeras cuatro olas de la ECHP, una considerando a todos los individuos presentes en estas cuatro olas, y otra considerando sólo a los individuos presentes en las ocho olas de la ECHP. Es decir, estiman una matriz que incorpora a todos los individuos encuestados en dos instancias sucesivas, y otra que elimina a los individuos que no fueron encuestados en aplicaciones posteriores de la encuesta. Si la atrición generara sesgos en las estimaciones de las matrices de transición, se esperaría observar diferencias importantes en los indicadores derivados de las dos matrices calculadas. Sin embargo, esto no ocurre: a pesar de que hay atrición selectiva, al parecer los índices calculados a partir de matrices de transición no son sensibles a este problema.

Por lo tanto este trabajo sigue a Ayala, Navarro y Sastre (2002) y estima matrices de transición que consideran, además de a los individuos presentes en encuestas sucesivas, matrices que incluyen únicamente a los individuos presentes en las tres aplicaciones de la Encuesta Panel Casen. Los resultados de estas estimaciones se presentan en las Tablas $N^{\text {os. }} 1,2$ y 3 . Éstas pueden compararse con las encontradas por Contreras, Cooper, Herman y Neilson (2004) y Castro (2008).

Estos datos de la Encuesta Panel Casen nos permiten estudiar el impacto de la atrición sobre las matrices de transición. En efecto, es posible comparar la matriz de transición del período 1996-2001, la cual contempla a todos los individuos encuestados en ambos años, con una matriz de transición para los mismos años que contempla sólo a los individuos que además están presentes en la encuesta 2006. Al mismo tiempo, es posible comparar la matriz de transición del período 20012006 con una matriz para los mismos años que contempla sólo a los individuos que además están presentes en la encuesta 1996. Como señalan Ayala, Navarro y Sastre (2002), de haber diferencias sustanciales entre matrices, habría evidencia a favor de la noción de que la atrición tiene un impacto importante, y habría que corregir por ella. Los resultados de este ejercicio de comparación, los cuales se presentan en la Tabla $\mathrm{N}^{\circ} 4$, muestran que las diferencias entre estas dos estimaciones son pequeñas (para todos los índices, salvo el B para el primer período). Esto refuta la noción de que la atrición tiene un impacto significativo sobre la estimación de la movilidad intrageneracional del ingreso para el caso de la Encuesta Panel Casen. 


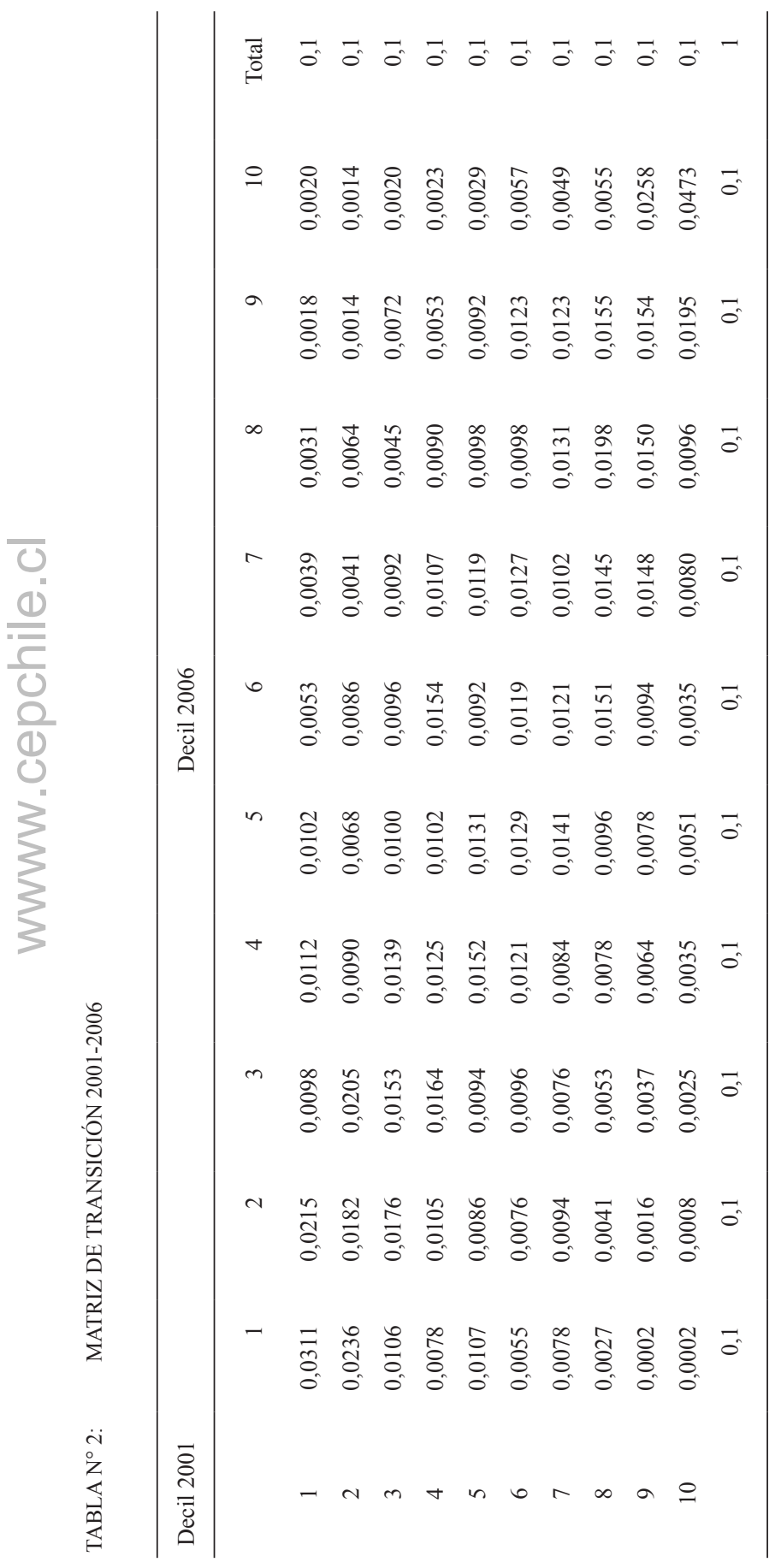




\begin{tabular}{|c|c|c|c|c|c|c|c|c|c|c|c|c|c|}
\hline & & 吾 & $\overrightarrow{0}$ & $\overrightarrow{0}$ & $\overrightarrow{0}$ & $\overrightarrow{0}$ & $\overrightarrow{0}$ & $\overrightarrow{0}$ & $\overrightarrow{0}$ & $\overrightarrow{0}$ & $\overrightarrow{0}$ & $\overrightarrow{0}$ & - \\
\hline & & $ㅇ$ & $\begin{array}{l}\hat{\widehat{\sigma}} \\
\text { ठ }\end{array}$ & $\begin{array}{l}\infty \\
\stackrel{8}{0} \\
0\end{array}$ & $\begin{array}{l}\vec{n} \\
\dot{8} \\
0\end{array}$ & $\begin{array}{l}\hat{o} \\
\hat{0} \\
0\end{array}$ & $\begin{array}{l}\text { to } \\
8 \\
0 \\
0\end{array}$ & $\begin{array}{l}\vec{\circ} \\
\vdots \\
\circ\end{array}$ & $\begin{array}{l}\frac{a}{0} \\
0 \\
0\end{array}$ & 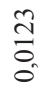 & $\begin{array}{l}\frac{0}{0} \\
\frac{0}{\mathrm{a}}\end{array}$ & $\begin{array}{l}\infty \\
\tilde{ల} \\
0 \\
0\end{array}$ & $\overrightarrow{0}$ \\
\hline & & $a$ & $\begin{array}{l}\text { fo } \\
8 \\
0\end{array}$ & $\begin{array}{l}\text { fo } \\
8 \\
0 \\
0\end{array}$ & $\begin{array}{l}\text { oे } \\
\dot{0} \\
0\end{array}$ & $\begin{array}{l}\tilde{n} \\
\tilde{\delta} \\
0 \\
0\end{array}$ & $\begin{array}{l}8 \\
\stackrel{0}{0} \\
0 \\
0\end{array}$ & $\frac{n}{0}$ & స్ & $\begin{array}{l}\text { ले } \\
0 \\
0\end{array}$ & 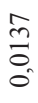 & $\frac{1}{\stackrel{0}{0}}$ & $\overrightarrow{0}$ \\
\hline & & $\infty$ & 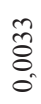 & $\begin{array}{l}0 \\
8 \\
8 \\
0 \\
0\end{array}$ & $\begin{array}{l}\frac{1}{\hat{8}} \\
\stackrel{8}{0} \\
0\end{array}$ & $\begin{array}{l}\stackrel{2}{0} \\
0 \\
0 \\
0\end{array}$ & $\begin{array}{l}+ \\
\text { Oे } \\
8 \\
0\end{array}$ & $\begin{array}{l}8 \\
\stackrel{0}{0} \\
0\end{array}$ & $\begin{array}{l}0 \\
\frac{n}{0} \\
0 \\
0\end{array}$ & $\begin{array}{l}\vec{N} \\
0 \\
0\end{array}$ & $\begin{array}{l}\text { to } \\
\text { : } \\
0\end{array}$ & $\begin{array}{l}8 \\
0 \\
0 \\
0\end{array}$ & $\overrightarrow{0}$ \\
\hline & & $r$ & $\begin{array}{l}\hat{0} \\
\tilde{\delta} \\
0\end{array}$ & $\begin{array}{l}\text { ळे } \\
\text { } \\
0\end{array}$ & $\begin{array}{l}\stackrel{2}{\circ} \\
\text { ठे } \\
0\end{array}$ & $\begin{array}{l}\text { ?a } \\
\text { वे } \\
0\end{array}$ & $\begin{array}{l}0 \\
\stackrel{8}{8} \\
0\end{array}$ & $\underset{\text { ले }}{\stackrel{0}{0}}$ & $\begin{array}{l}\stackrel{a}{\sigma} \\
0 \\
0\end{array}$ & $\begin{array}{l}\bar{n} \\
0 \\
0 \\
0\end{array}$ & 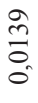 & $\begin{array}{l}\stackrel{2}{0} \\
0 \\
0\end{array}$ & $\overrightarrow{0}$ \\
\hline & 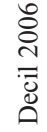 & 0 & ? & $\begin{array}{l}\infty \\
\stackrel{\infty}{0} \\
0 \\
0\end{array}$ & $\begin{array}{l}8 \\
\stackrel{8}{0} \\
0 \\
0\end{array}$ & $\begin{array}{l}\stackrel{\Xi}{\Xi} \\
0 \\
0\end{array}$ & 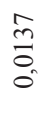 & $\begin{array}{l}\vec{m} \\
\stackrel{0}{0} \\
0\end{array}$ & $\begin{array}{l}\exists \\
\overline{0} \\
0\end{array}$ & $\begin{array}{l}\text { Õ } \\
0 \\
0 \\
0\end{array}$ & $\begin{array}{l}\hat{\delta} \\
\delta \\
\delta\end{array}$ & $\begin{array}{l}\tilde{8} \\
\tilde{\delta} \\
0\end{array}$ & $\overrightarrow{0}$ \\
\hline & & $n$ & 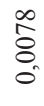 & $\begin{array}{l}\stackrel{a}{\vec{\sigma}} \\
0 \\
0\end{array}$ & $\begin{array}{l}\stackrel{8}{0} \\
8 \\
0 \\
0\end{array}$ & $\begin{array}{l}\hat{m} \\
\stackrel{0}{0} \\
0\end{array}$ & $\begin{array}{l}\vec{J} \\
\stackrel{\sigma}{0} \\
0\end{array}$ & $\begin{array}{l}8 \\
\stackrel{0}{0} \\
0\end{array}$ & 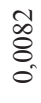 & $\begin{array}{l}\infty \\
\infty \\
8 \\
0\end{array}$ & 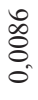 & 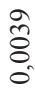 & $\overrightarrow{0}$ \\
\hline & & $\nabla$ & $\begin{array}{l}8 \\
8 \\
8 \\
0\end{array}$ & 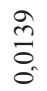 & $\begin{array}{l}\hat{\imath} \\
\stackrel{0}{0} \\
0\end{array}$ & $\begin{array}{l}\stackrel{8}{0} \\
\stackrel{0}{0}\end{array}$ & 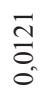 & $\begin{array}{l}\circ \\
8 \\
8 \\
0\end{array}$ & $\begin{array}{l}n \\
0 \\
0 \\
0\end{array}$ & $\begin{array}{l}+ \\
8 \\
8 \\
0\end{array}$ & $\begin{array}{l}: \\
\vdots \\
0 \\
0\end{array}$ & $\begin{array}{l}\bar{\Xi} \\
\stackrel{0}{\circ}\end{array}$ & $\overrightarrow{0}$ \\
\hline$\frac{\text { ò }}{z}$ & & $m$ & $\begin{array}{l}\text { o } \\
8 \\
0 \\
0\end{array}$ & $\begin{array}{l}\stackrel{2}{0} \\
0 \\
0\end{array}$ & $\begin{array}{l}\stackrel{0}{0} \\
0 \\
0 \\
0\end{array}$ & $\begin{array}{l}\tilde{2} \\
\stackrel{\tilde{0}}{0} \\
0\end{array}$ & $\begin{array}{l}\text { ô. } \\
\text { o. } \\
0\end{array}$ & $\begin{array}{l}\infty \\
\infty \\
0 \\
0 \\
0\end{array}$ & $\begin{array}{l}8 \\
\stackrel{0}{0} \\
0 \\
0\end{array}$ & $\begin{array}{l}1 \\
8 \\
8 \\
0\end{array}$ & $\begin{array}{l}\stackrel{2}{8} \\
8 \\
0 \\
0\end{array}$ & $\begin{array}{l}\vec{F} \\
8 \\
0 \\
0\end{array}$ & $\overrightarrow{0}$ \\
\hline $\begin{array}{l}\text { 离 } \\
\text { 㟧 }\end{array}$ & & $N$ & $\begin{array}{l}\text { ¿े } \\
\text { ¿े } \\
0\end{array}$ & $\begin{array}{l}\infty \\
0 \\
0 \\
0 \\
0\end{array}$ & $\begin{array}{l}\tilde{\overbrace{}} \\
\stackrel{0}{0}\end{array}$ & $\begin{array}{l}\stackrel{m}{0} \\
0 \\
0\end{array}$ & $\begin{array}{l}m \\
\stackrel{m}{0} \\
0 \\
0\end{array}$ & $\begin{array}{l}\bar{n} \\
\delta \\
0 \\
0\end{array}$ & 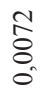 & $\begin{array}{l}n \\
\delta \\
\delta \\
0 \\
0\end{array}$ & $\begin{array}{l}\frac{1}{8} \\
\stackrel{0}{0} \\
0\end{array}$ & $\begin{array}{l}\tilde{\delta} \\
\stackrel{0}{0}\end{array}$ & $\overrightarrow{0}$ \\
\hline$\stackrel{\vec{E}}{\Sigma}$ & & - & $\begin{array}{l}\text { ¿े. } \\
\text { ठ̀ }\end{array}$ & $\begin{array}{l}\bar{a} \\
\overline{0} \\
0\end{array}$ & $\begin{array}{l}\stackrel{m}{0} \\
\stackrel{0}{0}\end{array}$ & $\begin{array}{l}\overrightarrow{\widetilde{I}} \\
0 \\
0\end{array}$ & $\begin{array}{l}n \\
\tilde{\delta} \\
8 \\
0\end{array}$ & $\begin{array}{l}\text { ڤे } \\
8 \\
0 \\
0\end{array}$ & $\begin{array}{l}n \\
\tilde{\delta} \\
0 \\
0\end{array}$ & $\begin{array}{l}\stackrel{+}{8} \\
8 \\
0\end{array}$ & 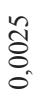 & 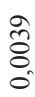 & $\overrightarrow{0}$ \\
\hline $\begin{array}{l}\ddot{m} \\
\stackrel{2}{Z} \\
\stackrel{n}{n}\end{array}$ & $\begin{array}{l}\text { ळे } \\
\overline{=} \\
\overline{0}\end{array}$ & & - & $N$ & $m$ & $\nabla$ & $n$ & 0 & $r$ & $\infty$ & $a$ & 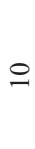 & 吾 \\
\hline
\end{tabular}


TABLA $N^{\circ} 4$ :

ESTUDIO DEL EFECTO DE LA ATRICIÓN SOBRE LOS ÍNDICES DE MOVILIDAD INTRAGENERACIONAL DEL INGRESO

\begin{tabular}{lccc}
\hline Período & B & S & I \\
\hline & & & \\
$1996-2001$ & 2,09 & 0,89 & 0,20 \\
$1996-2001$ (tres olas) & 1,93 & 0,87 & 0,22 \\
$2001-2006$ (tres olas) & 2,06 & 0,88 & 0,21 \\
$2001-2006$ & 0,87 & 0,22 \\
\hline
\end{tabular}

Se observa que en todas las matrices estimadas el décimo decil presenta la mayor proporción de miembros que no cambian de decil, con el 43,5\% en la transición 1996-2001, el 47\% en la transición 20012006, y el 37\% en la transición 1996-2001. Este dato es relevante, pues ha sido el fundamento de teorías que postulan que existe un proceso aleatorio distinto para el décimo decil. La siguiente sección intenta refutar esto, pero en el intertanto vale la pena mencionar que los individuos del décimo decil, al igual que los del primero, sólo pueden salir de su decil en una dirección. Esto implica que aquellos individuos que se encuentran inicialmente muy lejos del punto de corte (por debajo, en el caso del primer decil, y por arriba, en el caso del décimo) no se moverán de decil aunque enfrenten shocks significativos a su ingreso. Eso explica, al menos en parte, por qué el primero y el décimo decil presentan una probabilidad de no cambiar de decil sustancialmente mayor que la de los otros deciles. Adicionalmente, al comparar las Tablas $\mathrm{N}^{\text {os. }} 1 \mathrm{y}$ 2 con la 3 , se observa que la movilidad de la tercera es evidentemente mayor que la de las otras dos. Esto es razonable, pues se debería esperar mayor movilidad a medida que aumenta el tiempo entre mediciones; de hecho, dos matrices de transición con distintos horizontes temporales no son estrictamente comparables ${ }^{2}$.

A pesar de que es necesario tomar en cuenta el comentario final del párrafo anterior, es interesante hacer una comparación. La Tabla $\mathrm{N}^{\circ} 5$ compara los índices de Bartholomew, Shorrocks y el Immobility Ratio ${ }^{3}$

${ }^{2}$ A instancias de un referee anónimo se examinó hasta qué punto el proceso de cambio en el ingreso per cápita está generado por cambios en la composición del hogar en lugar de cambios en el ingreso. Se encontró que el análisis de varianza indica que sólo entre 4 y 5\% del cambio en el ingreso per cápita es por cambios en el número de integrantes del hogar.

${ }^{3}$ Para una descripción del cálculo de estos índices, véase el Apéndice. 
TABLA N ${ }^{\circ}$ 5: $\quad$ COMPARACIÓN INTERNACIONAL DE ÍNDICES DE MOVILIDAD INTRAGENERACIONAL DEL INGRESO

\begin{tabular}{|c|c|c|c|c|c|}
\hline \multirow[b]{2}{*}{ País } & \multirow[b]{2}{*}{ Período } & \multicolumn{3}{|c|}{ Estimadores } & \multirow[b]{2}{*}{ Fuente } \\
\hline & & B & $\mathrm{S}$ & I & \\
\hline \multicolumn{6}{|l|}{ Mediano plazo } \\
\hline \multirow[t]{2}{*}{ Chile } & 1996-2001 & 2,09 & 0,89 & 0,20 & Propias \\
\hline & 2001-2006 & 2,06 & 0,88 & 0,21 & Propias \\
\hline \multirow[t]{2}{*}{ Reino Unido } & 1993-1997 & 1,852 & 0,857 & 0,229 & Sastre y Ayala \\
\hline & 1994-1997 & 1,634 & 0,810 & 0,271 & Ayala, Navarro y Sastre \\
\hline \multirow[t]{2}{*}{ Francia } & 1993-1997 & 1,347 & 0,776 & 0,302 & Sastre y Ayala \\
\hline & 1994-1997 & 1,388 & 0,748 & 0,327 & Ayala, Navarro y Sastre \\
\hline \multirow[t]{2}{*}{ Alemania } & 1993-1997 & 1,760 & 0,803 & 0,277 & Sastre y Ayala \\
\hline & 1994-1997 & 1,649 & 0,789 & 0,290 & Ayala, Navarro y Sastre \\
\hline \multirow[t]{2}{*}{ Italia } & 1993-1997 & 1,729 & 0,831 & 0,252 & Sastre y Ayala \\
\hline & 1994-1997 & 1,721 & 0,808 & 0,273 & Ayala, Navarro y Sastre \\
\hline \multirow[t]{2}{*}{ España } & 1993-1997 & 1,775 & 0,845 & 0,240 & Sastre y Ayala \\
\hline & 1994-1997 & 1,572 & 0,780 & 0,298 & Ayala, Navarro y Sastre \\
\hline Estados Unidos & 1993-1997 & 1,667 & 0,818 & 0,264 & Sastre y Ayala \\
\hline \multicolumn{6}{|l|}{ Largo plazo } \\
\hline Chile & $1996-2006$ & 2,32 & 0,92 & 0,17 & Propias \\
\hline Italia & $1993-2000$ & 1,663 & 0,849 & 0,236 & Regoli, Quintano y Castellano \\
\hline
\end{tabular}

$\mathrm{B}=$ Índice de Bartholomew. $\mathrm{S}=$ Índice de Shorrocks. I = Immobility Ratio.

obtenidos para Chile con los obtenidos para otros países. Los índices para Chile son con intervalos de 5 años y los índices para los otros países son con intervalos de 3 y 4 años. La comparación de largo plazo con Italia utiliza un intervalo de 10 años para Chile y de 7 para Italia. En todos estos casos los intervalos son mayores para Chile y es de esperar que eso arroje mayor movilidad en Chile que la que se daría si los intervalos correspondieran exactamente a los de otros países. Si los índices de otros países fueran mayores que los de Chile, podría afirmarse, sin dudar, que Chile es menos móvil. Sin embargo, siendo los índices de Chile más altos, es factible que Chile sea más móvil (aunque no puede afirmarse con seguridad).

Los tres índices muestran que Chile, entre los años 1996-2001 y 2001-2006, tendría niveles de movilidad similares a los del Reino Unido para los años 1993-1997, y mayores que los de Francia, Alemania, 
Italia y España. Por ejemplo, el Índice de Bartholomew (B) para Chile es de 2,09 entre 1996 y 2001, y de 2,06 entre 2001 y 2006, valores que son mayores que los encontrados para el Reino Unido $(1,852)$, España $(1,775)$, Alemania $(1,760)$, Italia $(1,729)$, Estados Unidos $(1,667)$ y Francia $(1,347)$ entre 1993 y 1997. Estos resultados podrían ser evidencia de que Chile tiene una distribución del ingreso más móvil en términos intrageneracionales que la de los países antes mencionados, tema que requiere de mayor estudio para su validación. Sin embargo la presunción nuestra es que muchos autores habrían afirmado, antes de que se hiciera una comparación de este tipo, que la comparación entre Chile y estos países, aun con la diferencia de períodos, daría que Chile es más inmóvil.

Es importante señalar que los índices de movilidad intrageneracional del ingreso antes mencionados miden independencia intertemporal. Como ejemplo, supongamos que hay sólo dos categorías de ingreso posibles (bajo y alto) y consideremos las siguientes matrices de transición:

$$
\left[\begin{array}{ll}
0 & 1 \\
1 & 0
\end{array}\right] \quad\left[\begin{array}{ll}
0,5 & 0,5 \\
0,5 & 0,5
\end{array}\right]
$$

Los índices de movilidad antes mencionados postulan que la máxima movilidad es capturada por la matriz del lado derecho, que representa independencia temporal perfecta, mientras que alguien que postulara que lo deseable es exclusivamente reversal, señalaría que la matriz de movilidad máxima sería la de la izquierda. Los altos índices de Shorrocks, Bartholomew e Immobility Ratio estimados muestran que Chile presenta alta movilidad intrageneracional del ingreso si se busca independencia temporal. En este sentido, si lo que se valora es la independencia temporal, la movilidad estimada no puede interpretarse como vulnerabilidad. En tanto, si se busca reversal, se valoran matrices donde los individuos suben y bajan a lo largo de la distribución del ingreso, exactamente lo que se critica al señalar que la movilidad implica vulnerabilidad. Es decir, no tiene sentido referirse a la movilidad como vulnerabilidad si se busca reversal. Luego, bajo ninguna de las dos interpretaciones tendría sentido señalar que la movilidad intrageneracional del ingreso en Chile se encuentra en niveles indeseables.

En conclusión, esta sección encuentra que Chile presenta un nivel de movilidad intrageneracional del ingreso similar al de otros países, que la movilidad desde el décimo decil hacia otros deciles es más baja que la movilidad de cualquier otro decil hacia otros deciles, y que el problema de atrición sería de segundo orden para estas estimaciones. 
La siguiente sección estudia si los resultados encontrados para el último decil implican necesariamente que éste presenta una dinámica distinta a los demás deciles.

\section{2. ¿Un proceso distinto para el décimo decil?}

Las Tablas Nos. 1,2 y 3 muestran que en las tres transiciones estudiadas (1996-2001, 2001-2006 y 1996-2006) los individuos del décimo decil son los más propensos a mantenerse en su propio decil. Como se menciona anteriormente, es esperable encontrar que tanto el primero como el décimo decil exhiban una mayor propensión a no cambiar de decil, pues ambos tienen sólo una "vía de salida". Es decir, la mayor rigidez que se observa para estos deciles es, al menos en parte, producto de la herramienta que estamos utilizando para estudiar la movilidad del ingreso y no de algún fenómeno especial que ocurre en éstos.

A pesar de lo anterior, algunos autores han argumentado que los resultados obtenidos para el décimo decil implican que existen barreras a la movilidad desde y hacia dicho grupo. En concreto, Contreras, Cooper, Herman y Neilson (2004) postulan la existencia de barreras que bloquean la movilidad de los individuos del décimo decil. ¿Es realmente necesario que existan dichas barreras para explicar los resultados encontrados? El objetivo del ejercicio que se muestra a continuación es presentar un contrafactual que invalida la hipótesis de aquel trabajo. Un objetivo diferente, que no es el de este trabajo, sería encontrar el proceso aleatorio que mejor aproxima la generación de ingresos en Chile 4 .

Para estudiar esto se utilizan simulaciones de los cambios de los ingresos de los individuos presentes en las tres aplicaciones de la Encuesta Panel Casen. La metodología consiste en asignarle a cada individuo un shock porcentual a su ingreso aleatorio, sacado de una distribución $\mathrm{N}(0,2)$, truncada en $-1^{5}$. El resultado de estas simulaciones se presenta en las Tablas $N^{\text {os. }} 6$ y 7 , las cuales presentan las matrices de transición calculadas a partir de estas simulaciones, y las comparan con las matrices de transición efectivas.

${ }^{4}$ A instancias de un referee anónimo se hizo el intento de estimar este proceso por GMM. El proceso que se determina depende de los parámetros iniciales. A veces el proceso converge en $(0,2)$, otras veces no converge y a veces converge en un proceso diferente a $(0,2)$ pero nunca demasiado diferente.

${ }^{5}$ El truncamiento es necesario, pues el mínimo shock porcentual factible al ingreso es de $-100 \%$. Se realizaron mil simulaciones. La matriz a la que se converge después de mil simulaciones sólo difiere de aquella con 10 mil simulaciones en el cuarto decimal. 


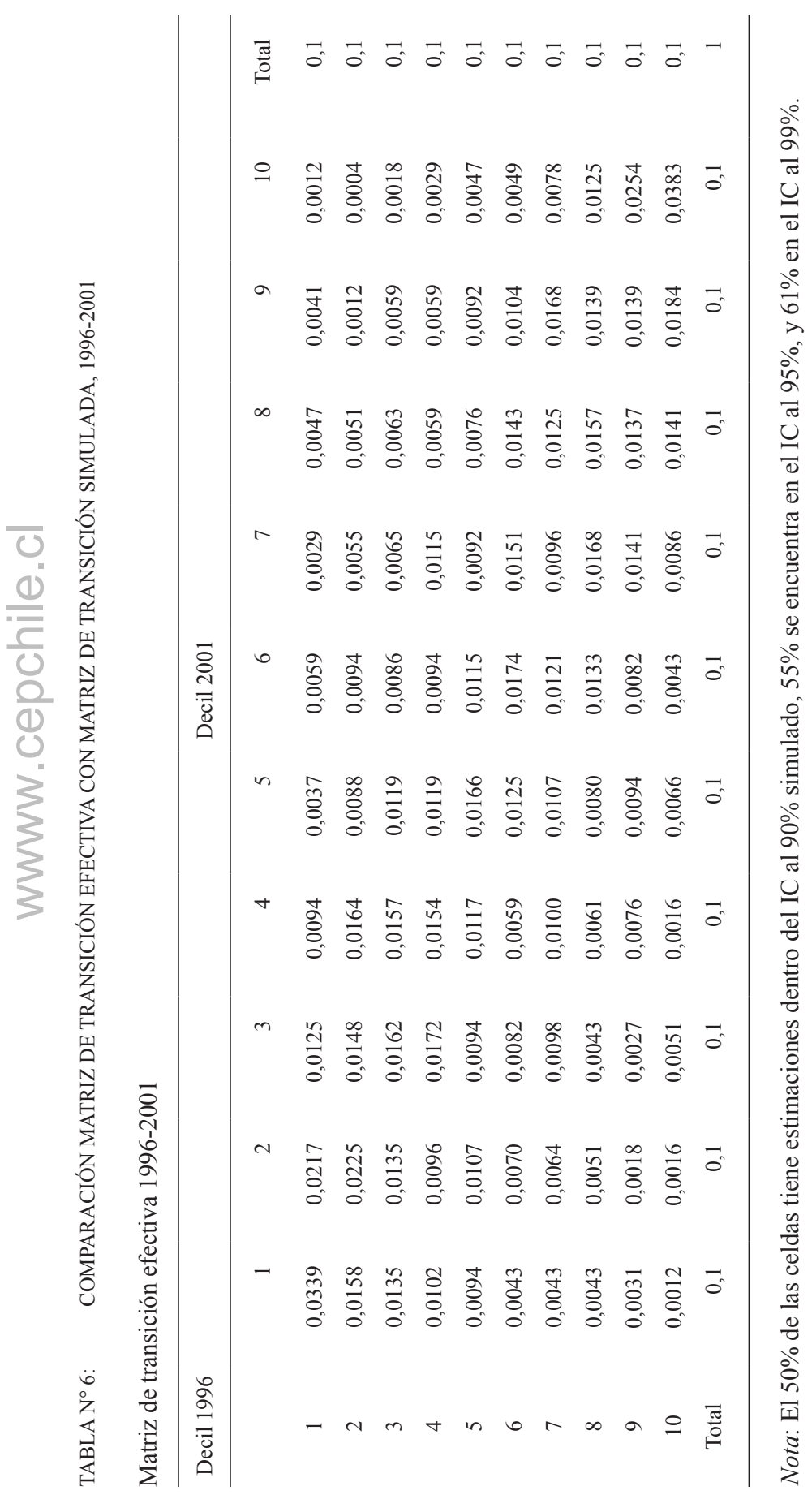




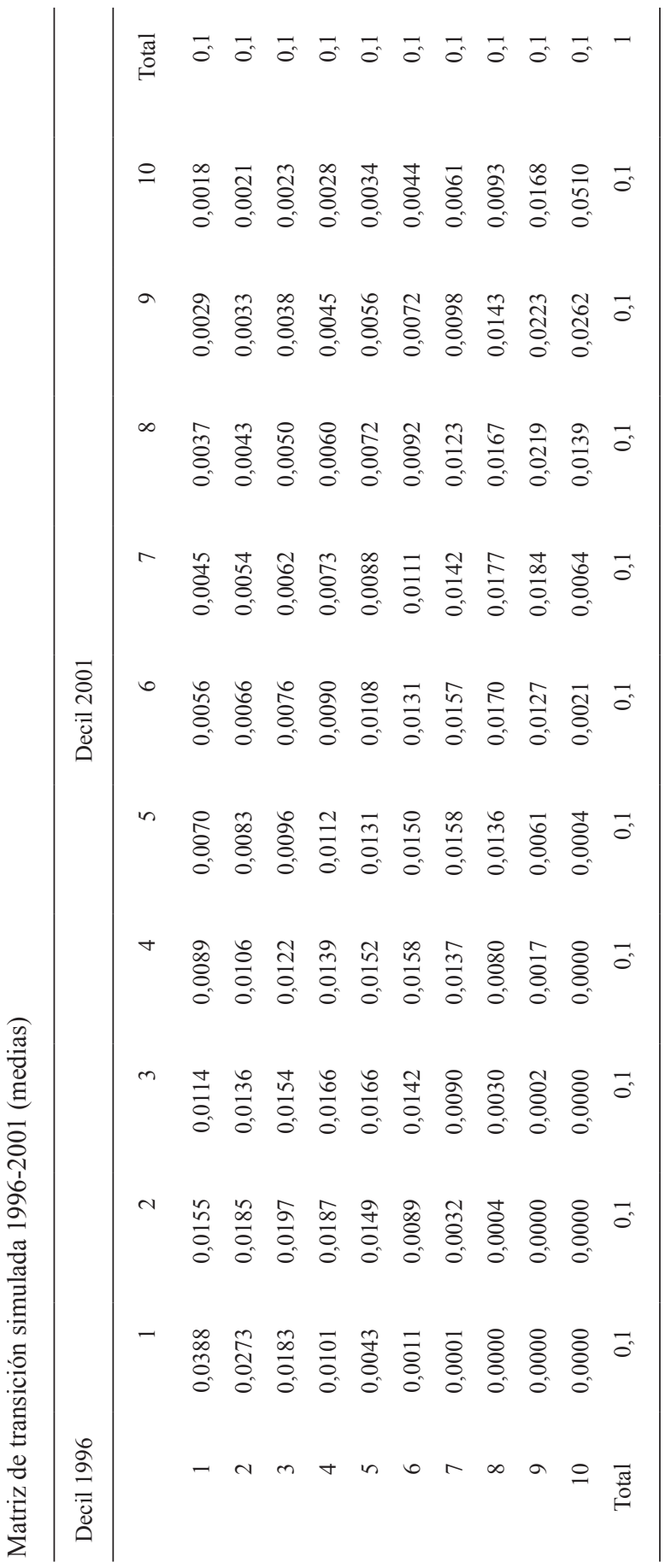




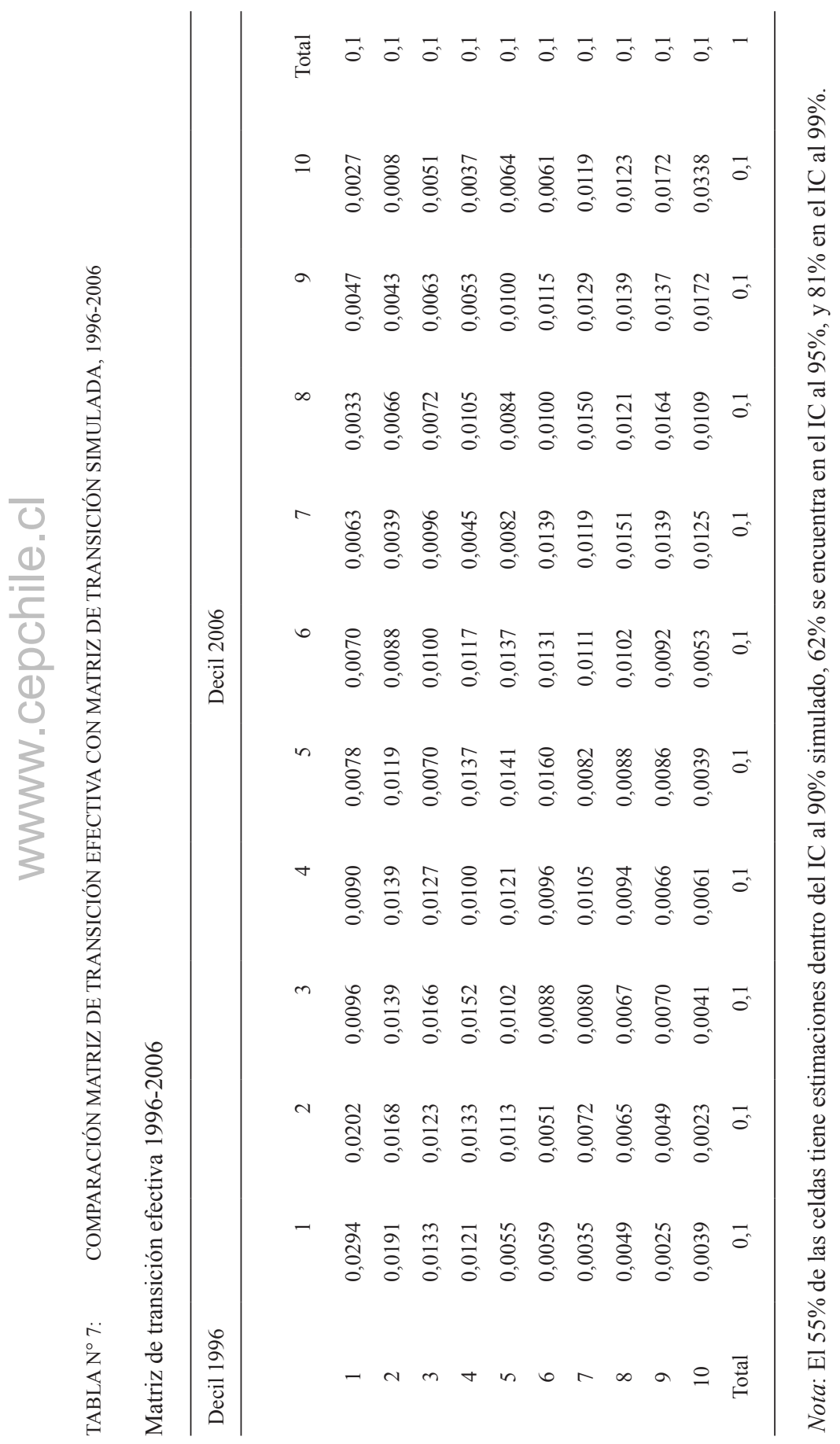




\begin{tabular}{|c|c|c|c|c|c|c|c|c|c|c|c|c|}
\hline & 苟 & $\overrightarrow{0}$ & $\overrightarrow{0}$ & $\overrightarrow{0}$ & $\overrightarrow{0}$ & $\overrightarrow{0}$ & $\overrightarrow{0}$ & $\overrightarrow{0}$ & $\overrightarrow{0}$ & $\overrightarrow{0}$ & $\overrightarrow{0}$ & - \\
\hline & 은 & $\begin{array}{l}\text { ¿े } \\
\text { ठ̀. } \\
\text { o. }\end{array}$ & $\begin{array}{l}\text { ल̂े } \\
\hat{\delta} \\
0\end{array}$ & 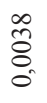 & $\begin{array}{l}\text { of } \\
8 \\
0 \\
0\end{array}$ & $\begin{array}{l}\tilde{2} \\
\tilde{\delta} \\
0 \\
0\end{array}$ & 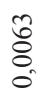 & $\begin{array}{l}\vec{\infty} \\
\stackrel{0}{0} \\
0\end{array}$ & $\begin{array}{l}\infty \\
\stackrel{0}{0} \\
0 \\
0\end{array}$ & $\begin{array}{l}n \\
\stackrel{0}{0} \\
0 \\
0\end{array}$ & 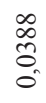 & $\overrightarrow{0}$ \\
\hline & $a$ & ڤે & $\begin{array}{l}\stackrel{\infty}{8} \\
\stackrel{0}{0} \\
0\end{array}$ & $\begin{array}{l}n \\
\tilde{\delta} \\
\delta \\
0\end{array}$ & 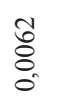 & $\begin{array}{l}\mathbb{2} \\
\stackrel{8}{8} \\
0\end{array}$ & $\begin{array}{l}\varkappa \\
\infty \\
0 \\
0 \\
0\end{array}$ & $\begin{array}{l}\text { to } \\
0 \\
0 \\
0\end{array}$ & $\begin{array}{l}\bar{m} \\
0 \\
0 \\
0\end{array}$ & $\begin{array}{l}\frac{n}{2} \\
\vdots \\
0\end{array}$ & 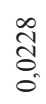 & $\overrightarrow{0}$ \\
\hline & $\infty$ & \begin{tabular}{l}
$\infty$ \\
\multirow{2}{*}{} \\
0 \\
0
\end{tabular} & $\begin{array}{l}\text { हे } \\
\text { ठे } \\
0\end{array}$ & $\begin{array}{l}\text { ஜ: } \\
\text { ठ } \\
0\end{array}$ & $\begin{array}{l}+t \\
\stackrel{+}{8} \\
0 \\
0\end{array}$ & $\begin{array}{l}\circ \\
\infty \\
8 \\
0 \\
0\end{array}$ & $\begin{array}{l}\hat{\alpha} \\
\hat{\delta} \\
0\end{array}$ & 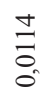 & $\begin{array}{l}\hat{n} \\
0 \\
0 \\
0\end{array}$ & \begin{tabular}{l}
$n$ \\
\multirow{0}{0}{} \\
0 \\
0
\end{tabular} & $\begin{array}{l}\stackrel{0}{2} \\
\stackrel{n}{0} \\
0\end{array}$ & $\overrightarrow{0}$ \\
\hline & $r$ & $\begin{array}{l}\hat{n} \\
8 \\
0 \\
0\end{array}$ & $\begin{array}{l}\text { ठे } \\
\text { ठ } \\
0\end{array}$ & 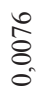 & $\begin{array}{l}+ \\
\infty \\
0 \\
0 \\
0\end{array}$ & $\begin{array}{l}\mathscr{2} \\
\stackrel{8}{0} \\
0\end{array}$ & $\begin{array}{l}\stackrel{0}{\circ} \\
0 \\
0\end{array}$ & $\underset{\Xi}{\tilde{O}}$ & 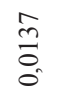 & $\begin{array}{l}\stackrel{\infty}{+} \\
\stackrel{0}{0}\end{array}$ & $\begin{array}{l}n \\
0 \\
0 \\
0\end{array}$ & $\overrightarrow{0}$ \\
\hline 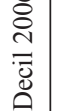 & 6 & \begin{tabular}{l}
$\infty$ \\
\multirow{8}{0}{} \\
0 \\
0
\end{tabular} & $\begin{array}{l}\hat{\widehat{\delta}} \\
\dot{0} \\
0\end{array}$ & $\begin{array}{l}\infty \\
\infty \\
8 \\
0 \\
0\end{array}$ & $\begin{array}{l}\text { oे } \\
\text { oे } \\
0\end{array}$ & $\begin{array}{l}n \\
0 \\
0 \\
0 \\
0\end{array}$ & $\begin{array}{l}0 \\
\stackrel{\sigma}{0} \\
0 \\
0\end{array}$ & 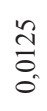 & $\begin{array}{l}\bar{m} \\
0 \\
0\end{array}$ & $\begin{array}{l}\hat{N} \\
0 \\
0 \\
0\end{array}$ & $\begin{array}{l}8 \\
8 \\
8 \\
0\end{array}$ & $\overrightarrow{0}$ \\
\hline & in & $\begin{array}{l}\vec{\infty} \\
\stackrel{8}{0} \\
0\end{array}$ & $\begin{array}{l}\text { to } \\
\text { \&े } \\
0\end{array}$ & $\begin{array}{l}\overline{0} \\
\stackrel{0}{\circ} \\
0\end{array}$ & $\begin{array}{l}\infty \\
\stackrel{0}{0} \\
0 \\
0\end{array}$ & $\begin{array}{l}\stackrel{n}{\Xi} \\
\sigma \\
0 \\
0\end{array}$ & $\begin{array}{l}\vec{\jmath} \\
0 \\
0\end{array}$ & $\begin{array}{l}\stackrel{J}{d} \\
0 \\
0\end{array}$ & $\begin{array}{l}\stackrel{1}{0} \\
0 \\
0\end{array}$ & $\begin{array}{l}\infty \\
\stackrel{8}{\delta} \\
0 \\
0\end{array}$ & $\begin{array}{l}n \\
\tilde{\delta} \\
0 \\
0\end{array}$ & $\overrightarrow{0}$ \\
\hline & $\nabla$ & $\begin{array}{l}\infty \\
\stackrel{0}{8} \\
\stackrel{0}{0}\end{array}$ & $\begin{array}{l}\Xi \\
\bar{\sigma} \\
0\end{array}$ & $\stackrel{\Xi}{\equiv}$ & $\underset{\tilde{0}}{\stackrel{0}{0}}$ & 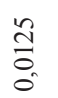 & $\begin{array}{l}\approx \\
\stackrel{a}{a} \\
0\end{array}$ & $\begin{array}{l}\stackrel{a}{\sigma} \\
\vdots \\
0\end{array}$ & $\begin{array}{l}\overrightarrow{0} \\
0 \\
0 \\
0\end{array}$ & $\begin{array}{l}\hat{8} \\
8 \\
0\end{array}$ & $\frac{n}{8}$ & $\overrightarrow{0}$ \\
\hline & $n$ & 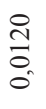 & $\begin{array}{l}\vec{m} \\
\stackrel{0}{0} \\
0\end{array}$ & $\begin{array}{l}\stackrel{n}{0} \\
\stackrel{0}{0} \\
0\end{array}$ & $\begin{array}{l}n \\
\stackrel{n}{0} \\
0 \\
0\end{array}$ & $\begin{array}{l}\vec{m} \\
\stackrel{0}{0}\end{array}$ & $\begin{array}{l}\tilde{I} \\
\stackrel{0}{0}\end{array}$ & $\begin{array}{l}n \\
0 \\
0 \\
0\end{array}$ & $\begin{array}{l}\infty \\
\frac{\infty}{8} \\
0\end{array}$ & $\begin{array}{l}\tilde{\delta} \\
\tilde{\delta}\end{array}$ & $\begin{array}{l}\text { oे } \\
\delta \\
0\end{array}$ & $\overrightarrow{0}$ \\
\hline & $N$ & $\begin{array}{l}\stackrel{0}{n} \\
\frac{n}{0} \\
0\end{array}$ & $\begin{array}{l}8 \\
\stackrel{8}{0} \\
0 \\
0\end{array}$ & $\begin{array}{l}\stackrel{0}{n} \\
\stackrel{0}{0} \\
0\end{array}$ & $\begin{array}{l}\hat{f} \\
0 \\
0 \\
0\end{array}$ & $\begin{array}{l}\stackrel{2}{\sigma} \\
\stackrel{0}{0} \\
0\end{array}$ & $\begin{array}{l}\stackrel{0}{\sigma} \\
\stackrel{0}{0} \\
\stackrel{0}{0}\end{array}$ & $\begin{array}{l}\vec{\infty} \\
\stackrel{8}{0} \\
0\end{array}$ & 守 & $\begin{array}{l}0 \\
8 \\
0\end{array}$ & 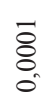 & $\overrightarrow{0}$ \\
\hline & - & $\frac{1}{0}$ & $\begin{array}{l}\infty \\
\stackrel{\infty}{\delta} \\
\delta_{0}\end{array}$ & $\begin{array}{l}8 \\
\stackrel{0}{0} \\
0 \\
0\end{array}$ & $\begin{array}{l}\stackrel{2}{I} \\
0 \\
0\end{array}$ & $\begin{array}{l}\circ \\
\& \\
0 \\
0 \\
0\end{array}$ & $\begin{array}{l}n \\
\tilde{\delta} \\
0\end{array}$ & $\underset{\hat{\sigma}}{\hat{\delta}}$ & $\begin{array}{l}0 \\
8 \\
8 \\
0\end{array}$ & $\begin{array}{l}\text { ఠ̊ } \\
\delta \\
\delta\end{array}$ & $\begin{array}{l}8 \\
8 \\
\vdots \\
0\end{array}$ & $\overrightarrow{0}$ \\
\hline $\begin{array}{l}2 \\
\stackrel{\Xi}{0} \\
\bar{\Xi} \\
0\end{array}$ & & - & $N$ & $m$ & $\nabla$ & in & 0 & $\pi$ & $\infty$ & $a$ & 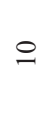 & 。ّٓ \\
\hline
\end{tabular}


La Tabla $\mathrm{N}^{\circ} 6$ muestra que para la transición 1996-2001 las simulaciones entregan una probabilidad de $38,8 \%$ de que un individuo del primer decil se mantenga en éste, mientras que la probabilidad efectiva es de 33,9\%. Al mismo tiempo, las simulaciones generan una probabilidad de $51 \%$ de que un individuo del décimo decil se mantenga en éste, mientras que la probabilidad efectiva es de 38,3\%. Es decir, suponiendo el mismo proceso aleatorio para todos los individuos encuestados podemos generar matrices de transición con mayor rigidez para el décimo decil que las obtenidas para Chile. El punto clave acá consiste en que un proceso aleatorio permite generar un similar número en la celda decil $1 /$ decil 1 y un número apreciablemente mayor en la celda decil 10/ decil 10. O sea, un proceso aleatorio sin ninguna rigidez para salir del decil 10 genera una rigidez aparente aún mayor en la matriz de transición. Con este contrafactual podemos afirmar que el número de la matriz de Chile no es de por sí un indicador de rigidez.

La Tabla $\mathrm{N}^{\circ} 7$ muestra el resultado del mismo ejercicio para la transición 1996-2006. En este caso, las simulaciones generan una probabilidad del $31,2 \%$ de que un individuo del primer decil se mantenga en éste, mientras que la probabilidad efectiva es de $29,4 \%$. En tanto, para el décimo decil las simulaciones predicen una probabilidad de no cambiar de decil del $38,8 \%$, mientras que la efectiva es de $33,8 \%$. Nuevamente se observa que mediante un proceso aleatorio igual para todos los individuos de la sociedad es posible generar matrices de transición con mayor rigidez para el décimo decil que la observada en Chile.

Es importante mencionar que las matrices simuladas presentan índices de movilidad similares a los observados en Chile, como muestra la Tabla $\mathrm{N}^{\circ}$ 8. En efecto, las simulaciones generan matrices con los mismos índices de Shorrocks e Immobility Ratio que los efectivos. Esto muestra que las simulaciones permiten generar matrices de transición muy similares a las obtenidas para Chile, e incluso con mayor rigidez para el décimo decil, sin que existan barreras que bloqueen la movilidad desde o hacia dicho decil.

En conclusión, la existencia de barreras a la movilidad del décimo decil no es necesaria para explicar la evidencia encontrada para Chile. Por el contrario, esta evidencia surge por la existencia de individuos que están muy lejos del punto de corte. En el caso de Chile, como la cola derecha de la distribución del ingreso es tan larga, eso por sí mismo genera alto porcentaje de inmovilismo en el décimo 


\begin{tabular}{lccc}
\hline Índices & S & B & I \\
\hline Chile 1996-2001 & 0,89 & 2,09 & 0,20 \\
Simulación 1996-2001 & 0,87 & 1,81 & 0,22 \\
Chile 1996-2006 & 0,92 & 2,32 & 0,17 \\
Simulación 1996-2001 & 0,91 & 2,21 & 0,18 \\
\hline
\end{tabular}

decil, pero eso, como se demostró, parece ser una característica de la distribución del ingreso más que de la naturaleza del proceso de movilidad de ingresos.

\section{Movilidad intergeneracional del ingreso}

A diferencia de la movilidad intrageneracional del ingreso, que resume los movimientos a lo largo de la distribución del ingreso para los mismos individuos en períodos relativamente cortos, la movilidad intergeneracional del ingreso estudia la transferencia de riqueza entre generaciones, con el objetivo de determinar en qué grado los ingresos de los hijos están determinados por los de sus padres. En este caso, sociedades con menor movilidad intergeneracional del ingreso son más rígidas y presentan menos oportunidades para surgir. Hay consenso en la literatura en torno a la noción de que mayor movilidad en este plano es deseable.

En Chile se ha afirmado que la movilidad intrageneracional es demasiado alta y la intergeneracional demasiado baja. Esa es la hipótesis de que Chile sería el "peor de los mundos", como se describió anteriormente. Dicha hipótesis consiste en considerar que un nivel alto de movilidad intergeneracional del ingreso, junto con un nivel bajo de movilidad intrageneracional, representan una situación donde los individuos no sólo sufren vaivenes aleatorios en sus ingresos durante sus vidas, sino que además el nivel en torno al cual éstos fluctúan está determinado principalmente por el ingreso de sus padres.

Ya se ha discutido que considerar la alta movilidad intrageneracional del ingreso como vulnerabilidad pareciera contradecir la noción de que una sociedad rígida en la que los ingresos no se mueven (no hay reversal ni independencia) es algo indeseable. El propósito de 
esta sección es discutir la segunda parte de la aseveración de que Chile es "el peor de los mundos", o sea las estimaciones de la movilidad intergeneracional del ingreso utilizadas para hacer esta aseveración, con el objetivo de precisar la visión sobre la dinámica del ingreso en Chile.

Las estimaciones de la movilidad intergeneracional del ingreso más comentadas han sido realizadas por Núñez y Risco (2004) y Núñez y Miranda (2006), quienes concluyen mediante comparaciones internacionales que Chile presenta niveles bajos de movilidad intergeneracional del ingreso. Sin embargo, la estimación de la movilidad intergeneracional del ingreso presenta una serie de problemas econométricos complejos, los cuales sesgan los resultados y dificultan la comparación internacional de los mismos. Esta sección presenta algunos de estos problemas y argumenta que la movilidad intergeneracional de la educación es una mejor medida de la movilidad social entre generaciones.

Los trabajos que estudian la movilidad intergeneracional del ingreso estiman mediante diversos métodos estadísticos la siguiente regresión:

$$
\mathrm{y}_{1 i}=\rho y_{0 i}+\mathrm{X}_{i}^{\prime} \beta+\varepsilon_{\mathrm{i}}
$$

Donde $\mathrm{y}_{1 \mathrm{i}}$ es alguna medida del ingreso del hijo, $\mathrm{y}_{0 \mathrm{i}}$ es alguna medida del ingreso de su padre, y $\mathrm{X}_{\mathrm{i}}$ es un vector de características del hijo por las cuales se desea controlar. ¿Cuál es la medida de ingreso apropiada? Es sabido que los ingresos presentan fluctuaciones significativas en el tiempo, y a raíz de esto se postula que los individuos toman decisiones de consumo en base a su ingreso permanente. Por ende, las medidas de ingreso relevantes serían el ingreso permanente de padres e hijos. Lamentablemente, no existen mediciones de dicha variable, por lo que la mayoría de los trabajos utiliza como proxy de ésta el ingreso corriente de cada individuo. Como es sabido, en la medida que este proxy exhiba error de medición clásico, el uso del ingreso corriente para el ingreso de los padres genera sesgo de atenuación, mientras que el uso del ingreso corriente de los hijos no genera sesgo, sino sólo ineficiencia ${ }^{6}$.

${ }^{6}$ Una buena explicación de esto se encuentra en Hausman (2001). 
Las estimaciones de la movilidad intergeneracional del ingreso adolecen de sesgo de atenuación, y la magnitud de dicho sesgo difiere entre países y muestras. Por ende, el ejercicio de estimar la movilidad intergeneracional del ingreso no aporta muchas luces sobre el grado de rigidez intergeneracional del ingreso en Chile en relación con otros países.

¿Es posible obtener una mejor aproximación a este fenómeno? Se necesitaría una medida de movilidad intergeneracional de alguna variable que estuviese fuertemente correlacionada con el ingreso y que no presentara los errores de medición antes mencionados. En este sentido, la movilidad intergeneracional de la educación cumple precisamente con estos dos criterios, ya que es claro que hay una relación entre nivel de educación e ingresos, y además las medidas de educación plausiblemente adolecen de menos error de medición que el incurrido al utilizar el ingreso corriente como proxy del ingreso permanente ${ }^{7}$.

La Tabla $\mathrm{N}^{\circ} 9$ presenta diferentes medidas de la movilidad intergeneracional de la educación, como el coeficiente de la educación del padre en una regresión de la educación del hijo sobre dicha variable y otros controles, y la tasa de retorno a la educación terciaria. En cuanto a la primera medida, se observa que Chile es un país relativamente móvil, con coeficientes entre 0,5 y 0,6 , similares a los de Suecia $(0,58)$ y Dinamarca $(0,49)$, y menores que los del Reino Unido $(0,71)$. A la vez, Sapelli (2008) encuentra que dicho coeficiente ha ido disminuyendo en el tiempo, lo que implica que la movilidad intergeneracional de la educación ha estado aumentando.

En resumen, se ha argumentado que la evidencia a favor de la noción de que Chile es un país con bajos niveles de movilidad intergeneracional del ingreso no es concluyente, puesto que las comparaciones directas con otros países no son permisibles por los sesgos previamente mencionados. Al mismo tiempo, la mejor aproximación al problema, la movilidad intergeneracional de la educación, muestra a Chile en un nivel similar al del resto del mundo. Esto sugiere que la segunda mitad de la hipótesis del "peor de los mundos" no tiene evidencia empírica concluyente a su favor.

${ }^{7}$ De hecho, aunque tuvieran error de medición significativo, en la medida que dicho error no difiera sistemáticamente entre países, las comparaciones internacionales seguirían siendo válidas. 
TABLA N ${ }^{\circ}$ 9: $\quad$ MEDIDAS DE MOVILIDAD INTERGENERACIONAL DEL INGRESO

\begin{tabular}{|c|c|c|c|c|c|c|c|}
\hline & Chile-Sapelli & Chile - Otros & UK & Francia & Alemania & Italia & España \\
\hline $\begin{array}{l}\text { Movilidad } \\
\text { intragene- } \\
\text { racional } \\
\text { del ingreso }\end{array}$ & $1,92(1)$ & & $1,85(2)$ & $1,35(2)$ & $1,76(2)$ & $1,73(2)$ & $1,78(2)$ \\
\hline $\begin{array}{l}\text { Coeficiente } \\
\text { educación } \\
\text { del padre }\end{array}$ & 0,49 (4) & $\begin{array}{l}0,43-0,55(5) \\
0,58-0,67(6) \\
0,52-0,54(7)\end{array}$ & $0,71(3)$ & & & 0,67 (3) & \\
\hline $\begin{array}{l}\text { Movilidad } \\
\text { intragene- } \\
\text { racional } \\
\text { del ingreso }\end{array}$ & Dinamarca & Australia & Noruega & Finlandia & Canadá & Suecia & EE. UU. \\
\hline $\begin{array}{l}\text { Coeficiente } \\
\text { educación } \\
\text { del padre }\end{array}$ & 0,49 (3) & & $0,4(3)$ & $0,48(3)$ & & $0,58(3)$ & $0,46(3)$ \\
\hline
\end{tabular}

Fuentes:

(1) Elaboración propia.

(2) Sastre y Ayala (2002).

(3) Hertz et al. (2007).

(4) Sapelli (2008).

(5) Núñez y Risco (2004).

(6) Contreras, Fuenzalida y Núñez (2006).

(7) Núñez y Miranda (2006).

\section{Análisis de los cambios en los ingresos Casen 1996-2001-2006}

La discusión anterior se ha centrado en analizar los trabajos que afirman que Chile está en "el peor de los mundos" en cuanto a movilidad. Se han discutido los tres principales argumentos que sustentan esta visión: la noción de movilidad como vulnerabilidad, la posible existencia de un proceso diferente para los individuos más ricos, y la idea de que Chile presenta alta rigidez intergeneracional del ingreso. Esta sección, en tanto, busca caracterizar los cambios en los ingresos que generan las matrices de transición observadas.

Con este objetivo, la Tabla $\mathrm{N}^{\circ} 10$ presenta el porcentaje de individuos cuyos ingresos reales aumentaron, se mantuvieron, o disminuyeron en las transiciones 1996-2001, 2001-2006, y 1996-2006. Para construirla se han utilizado las tres aplicaciones de la Encuesta Panel 
TABLA N ${ }^{\circ}$ 10: $\quad$ CAMBIO EN LOS INGRESOS INDIVIDUALES, TRANSICIONES 1996-2001, 2001-2006 Y 1996-2006

\begin{tabular}{lcccc}
\hline Definición de ingreso & Cambio & $1996-2001$ & $2001-2006$ & $1996-2006$ \\
\hline \multirow{3}{*}{ Ingreso total } & Aumenta & $63,0 \%$ & $51,1 \%$ & $66,6 \%$ \\
& Sin cambio & $4,8 \%$ & $10,6 \%$ & $4,8 \%$ \\
& Disminuye & $32,2 \%$ & $38,3 \%$ & $28,6 \%$ \\
\hline \multirow{3}{*}{ Ingreso total del hogar } & Aumenta & $59,5 \%$ & $54,3 \%$ & $63,2 \%$ \\
& Sin cambio & $6,3 \%$ & $6,9 \%$ & $5,8 \%$ \\
& Disminuye & $34,3 \%$ & $38,7 \%$ & $30,9 \%$ \\
\hline \multirow{3}{*}{ Ingreso del trabajo } & Aumenta & $62,3 \%$ & $53,1 \%$ & $67,2 \%$ \\
& Sin cambio & $5,4 \%$ & $9,3 \%$ & $4,2 \%$ \\
& Disminuye & $32,4 \%$ & $37,6 \%$ & $28,6 \%$ \\
\hline \multirow{3}{*}{ Ingreso del trabajo del hogar } & Aumenta & $55,9 \%$ & $55,8 \%$ & $61,6 \%$ \\
& Sin cambio & $5,0 \%$ & $6,6 \%$ & $4,6 \%$ \\
& Disminuye & $39,1 \%$ & $37,6 \%$ & $33,9 \%$ \\
\hline
\end{tabular}

Casen, eliminando a los individuos que no reportan ingresos ${ }^{8}$ y a aquellos en el $1 \%$ superior y $1 \%$ inferior de ingresos y de cambios en ingresos. Además, se ha definido como un aumento en los ingresos reales un alza superior al $5 \%$ en éstos, y como una caída una disminución de 5\% o más. Finalmente, se usan cuatro medidas de ingreso diferentes como ejercicio de robustez. Este ejercicio muestra que en las tres transiciones observadas el porcentaje de individuos cuyos ingresos aumentaron supera ampliamente al porcentaje de individuos cuyos ingresos cayeron, algo esperable en un país con crecimiento económico. De hecho, cuando el país creció menos, la movilidad fue más baja. Este resultado muestra que la movilidad intrageneracional observada es generada en buena parte por aumentos en los ingresos de los individuos, los cuales ocasionan nuevos movimientos en la distribución del ingreso.

Sin embargo, es posible que aquellos individuos cuyos ingresos aumentaron en la primera transición tengan una mayor probabilidad de sufrir caídas en el ingreso en su segunda transición. Este fenómeno, conocido como regresión a la media, implicaría que los movimientos a lo largo de la distribución del ingreso son transitorios, aumentando la movilidad medida como reversal pero no aumentando necesariamente el ingreso permanente de los individuos. Para estudiar esto se toman los tres desenlaces definidos anteriormente (Aumento, Sin Cambio, y

${ }^{8}$ Se elimina a aquellos individuos cuyo ingreso es cero o 9999999, y a aquellos cuyo ingreso no se reporta. 
Disminución) y se calculan las probabilidades de que cada evento ocurra en la transición 2001-2006, condicional en el desenlace ocurrido en la transición 1996-2001. El resultado de este ejercicio se presenta en la Tabla $\mathrm{N}^{\circ} 11$, donde la primera fila estudia a aquellos individuos cuyo ingreso real aumentó entre 1996 y 2001, y muestra la probabilidad de que su ingreso haya aumentado $(41,4 \%)$, caído $(45,4 \%)$, o se haya mantenido (12,8\%) entre 2001 y 2006 . La evidencia muestra que un aumento en los ingresos entre 2001 y 2006 es mucho más probable si se experimentó una caída de los ingresos entre 1996 y 2001 que si los ingresos aumentaron en dicho período (68,9\% vs. 41,4\%). Al mismo tiempo, se observa que quien ha tenido un aumento en el ingreso para la transición 1996-2001 tiene una probabilidad similar de que sus ingresos aumenten o se mantengan $(54,2 \%)$ a que éstos caigan $(45,4 \%)$. La combinación de, por un lado, una alta probabilidad de que aumenten los ingresos - condicional en una caída en el período anterior - con, por el otro, una alta probabilidad de que caigan los ingresos - condicional en un aumento en el período anterior-, es evidencia en favor de la existencia de regresión a la media. En este caso, sin embargo, si bien lo primero se cumple con claridad, lo segundo es menos claro.

Otra posible aproximación a este fenómeno sería estudiar regresiones de los cambios en los ingresos observados en variables explicativas sugeridas por la teoría, como el nivel de ingresos, la edad, y la escolaridad. La Tabla $\mathrm{N}^{\circ} 12$ presenta los resultados de este ejercicio. Para estimar estas regresiones se utilizan los ingresos de todos los individuos que reportan ingresos mayores que cero, y se elimina a aquellos individuos en el $1 \%$ más alto y en el $1 \%$ más bajo del nivel de ingresos y de cambio en los ingresos. Los resultados muestran un impacto negativo y significativo del nivel de ingresos sobre el cambio de los mismos, un impacto significativo y positivo de la edad y de los años de escolari$\mathrm{dad}^{9}$, y un impacto positivo de vivir en una zona urbana. Mientras que los resultados para edad y escolaridad concuerdan con lo esperado, los resultados para las zonas urbanas podrían reflejar una mayor disponibilidad de oportunidades en ellas. En tanto, los resultados para el nivel de ingreso apuntan a la misma idea que la evidencia aportada por las probabilidades condicionales: a menor ingreso en período base, mayor aumento de los ingresos en el siguiente período.

${ }^{9}$ Para el rango relevante de edades y de escolaridad. Mientras la primera se hace negativa para valores mayores que 100 en todas las regresiones, la segunda lo hace para valores mayores que 70 . 
TABLA N ${ }^{\circ}$ 11: $\quad$ CAMBIO EN LOS INGRESOS INDIVIDUALES, TRANSICIÓN 1996-2001 Y 2001-2006

\begin{tabular}{|c|c|c|c|c|}
\hline \multirow{3}{*}{ Ingreso total } & & $2001-2006$ & & \\
\hline & & Aumenta & Sin cambio & Disminuye \\
\hline & Aumenta & $41,4 \%$ & $12,8 \%$ & $45,8 \%$ \\
\hline \multirow[t]{2}{*}{$1996-2001$} & Sin cambio & $60,2 \%$ & $10,2 \%$ & $29,6 \%$ \\
\hline & Disminuye & $68,9 \%$ & $6,4 \%$ & $24,7 \%$ \\
\hline \multirow{3}{*}{ Ingreso total del hogar } & & $2001-2006$ & & \\
\hline & & Aumenta & Sin cambio & Disminuye \\
\hline & Aumenta & $47,2 \%$ & $6,4 \%$ & $46,4 \%$ \\
\hline \multirow[t]{2}{*}{$1996-2001$} & Sin cambio & $56,4 \%$ & $7,1 \%$ & $36,4 \%$ \\
\hline & Disminuye & $66,9 \%$ & $7,9 \%$ & $25,2 \%$ \\
\hline \multirow{3}{*}{ Ingreso del trabajo } & & $2001-2006$ & & \\
\hline & & Aumenta & Sin cambio & Disminuye \\
\hline & Aumenta & $43,0 \%$ & $11,0 \%$ & $46,0 \%$ \\
\hline \multirow[t]{2}{*}{ 1996-2001 } & Sin cambio & $66,0 \%$ & $6,0 \%$ & $28,0 \%$ \\
\hline & Disminuye & $70,4 \%$ & $6,7 \%$ & $22,9 \%$ \\
\hline \multirow{3}{*}{ Ingreso del trabajo del hogar } & & $2001-2006$ & & \\
\hline & & Aumenta & Sin cambio & Disminuye \\
\hline & Aumenta & $47,1 \%$ & $6,4 \%$ & $46,5 \%$ \\
\hline \multirow{2}{*}{$1996-2001$} & Sin cambio & $64,4 \%$ & $7,9 \%$ & $27,7 \%$ \\
\hline & Disminuye & $67,3 \%$ & $6,7 \%$ & $26,0 \%$ \\
\hline
\end{tabular}

Sin embargo, esta evidencia presenta ciertos matices interesantes. Al utilizar como especificación alternativa variables dummy representando el decil al que pertenece el individuo, se observa que el impacto del decil sobre el ingreso es similar para los primeros deciles, y se hace mayor sólo para los deciles más altos de la distribución. Esta evidencia se presenta en la Tabla $\mathrm{N}^{\circ} 13$ y en las Figuras $\mathrm{N}^{\text {os. }} 1,2$ y 3 . Es decir, se observa que las personas con mayores ingresos son más propensas, manteniendo todo lo demás constante, a que sus ingresos disminuyan. Esto es evidencia a favor de regresión a la media para los deciles más altos, y contrasta fuertemente con la noción de que la distribución del ingreso es más rígida intertemporalmente para los deciles más ricos.

Un posible problema con las regresiones anteriores es la existencia de error de medición en la variable ingreso. Como muestra Gottschalk (2005), los datos de ingreso obtenidos de encuestas presentan importantes errores de medición, lo que genera estimadores atenuados. Por ende, es posible que el impacto del ingreso sobre los cambios en 


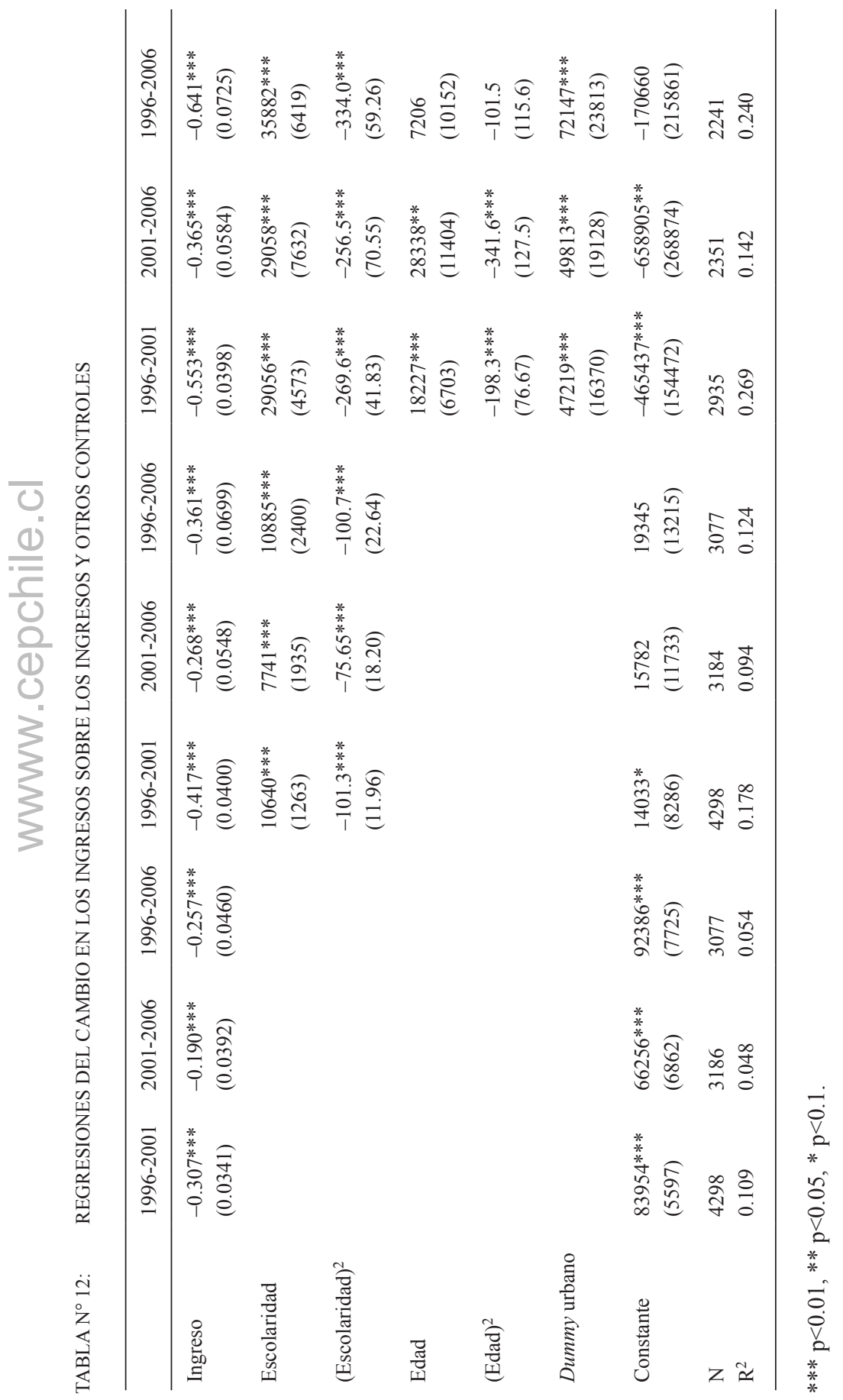


TABLA N ${ }^{\circ}$ 13: REGRESIÓN DEL CAMBIO EN LOS INGRESOS SOBRE EL DECIL DE LA DISTRIBUCIÓN DEL INGRESO Y OTROS CONTROLES

\begin{tabular}{|c|c|c|c|}
\hline & $1996-2001$ & 2001-2006 & $1996-2006$ \\
\hline \multirow[t]{2}{*}{ Escolaridad } & $9941 * * *$ & $7521 * * *$ & $10858 * * *$ \\
\hline & $(1100)$ & (1774) & $(2122)$ \\
\hline \multirow[t]{2}{*}{ Escolaridad al cuadrado } & $-94.21 * * *$ & $-73.70 * * *$ & $-100.0 * * *$ \\
\hline & $(10.45)$ & $(16.63)$ & $(20.11)$ \\
\hline \multirow[t]{2}{*}{ Segundo decil } & $-34583 * * *$ & $-15613^{*}$ & $-35899 * * *$ \\
\hline & $(10153)$ & $(8743)$ & (10548) \\
\hline \multirow[t]{2}{*}{ Tercer decil } & $-47717 * * *$ & -14945 & $-58793 * * *$ \\
\hline & $(10881)$ & (12798) & (11314) \\
\hline \multirow[t]{2}{*}{ Cuarto decil } & $-47538 * * *$ & $-26443 * *$ & $-48748 * * *$ \\
\hline & $(10856)$ & (12879) & $(13351)$ \\
\hline \multirow[t]{2}{*}{ Quinto decil } & $-40206 * * *$ & $-42541 * * *$ & $-71416^{* * *}$ \\
\hline & $(14654)$ & (9233) & (10471) \\
\hline \multirow[t]{2}{*}{ Sexto decil } & $-81635 * * *$ & -12718 & $-77993 * * *$ \\
\hline & $(12151)$ & $(17715)$ & (11410) \\
\hline \multirow[t]{2}{*}{ Séptimo decil } & $-72512^{* * *}$ & $-62561 * * *$ & $-40857 *$ \\
\hline & (11934) & $(11520)$ & $(24654)$ \\
\hline \multirow[t]{2}{*}{ Octavo decil } & $-86932 * * *$ & $-59131 * * *$ & $-85957 * * *$ \\
\hline & (13778) & (13379) & (17962) \\
\hline \multirow[t]{2}{*}{ Noveno decil } & $-116684^{* * * *}$ & $-66375^{* * *}$ & $-84532 * * *$ \\
\hline & (14526) & $(24124)$ & $(21493)$ \\
\hline \multirow[t]{2}{*}{ Décimo decil } & $-170535^{* * *}$ & $-107749 * * *$ & $-174239 * * *$ \\
\hline & $(17829)$ & $(20001)$ & $(20431)$ \\
\hline \multirow[t]{2}{*}{ Constante } & $27014 * * *$ & 17217 & $40549 * *$ \\
\hline & $(9649)$ & (11344) & $(15966)$ \\
\hline $\mathrm{N}$ & 4298 & 3184 & 3077 \\
\hline $\mathrm{R}^{2}$ & 0.146 & 0.084 & 0.145 \\
\hline
\end{tabular}

$* * * \mathrm{p}<0.01, * * \mathrm{p}<0.05, * \mathrm{p}<0.1$.

Errores estándares robustos entre paréntesis.

el mismo sea mayor, en valor absoluto, que el estimado. Con el objetivo de corroborar esto, se estimaron las regresiones de la Tabla $\mathrm{N}^{\circ} 13$ utilizando como instrumentos del ingreso el total de dormitorios de la vivienda en una especificación, y el total de dormitorios de la vivienda en otra. Los resultados de este ejercicio (no reportados) son coeficientes más cercanos a cero, en valor absoluto, que los de las regresiones originales, lo que podría ser interpretado como evidencia en contra de la importancia del error de medición en estas estimaciones, o como evidencia de que los instrumentos utilizados son pobres. En cualquier caso, aun con error de medición, la evidencia apunta a que los individuos con mayores ingresos tienen menores cambios en el ingreso. 
FIGURA N ${ }^{\circ}$ 1: $\quad$ EFECTO DEL DECIL SOBRE EL CAMBIO DEL INGRESO, TRANSICIÓN 1996-2001

1996-2001

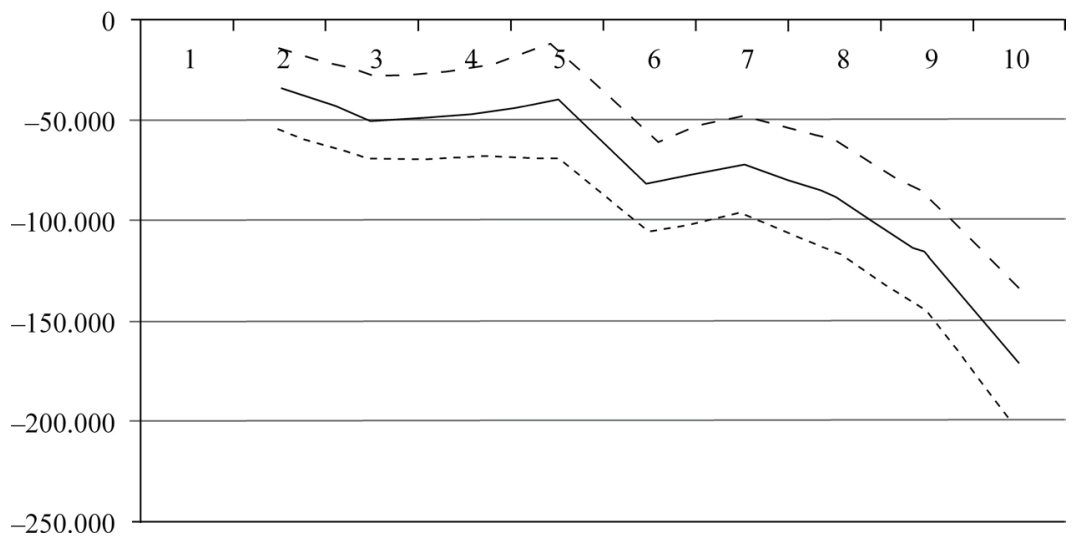

FIGURA N ${ }^{\circ}$ 2: $\quad$ EFECTO DEL DECIL SOBRE EL CAMBIO DEL INGRESO, TRANSICIÓN 2001-2006

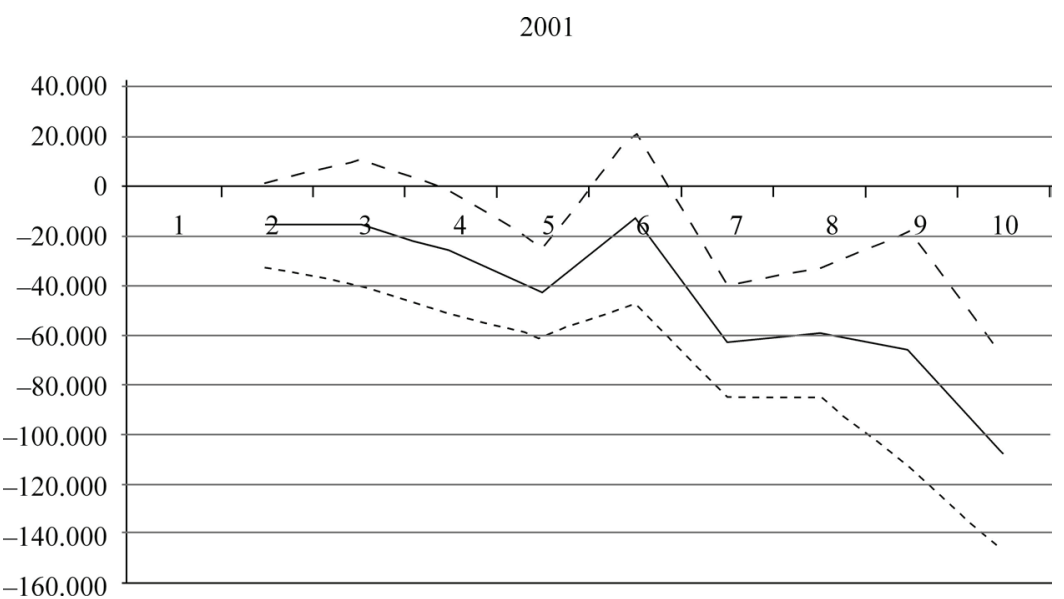


FIGURA N ${ }^{\circ}$ 3: $\quad$ EFECTO DEL DECIL SOBRE EL CAMBIO DEL INGRESO, TRANSICIÓN 1996-2006

1996-2006

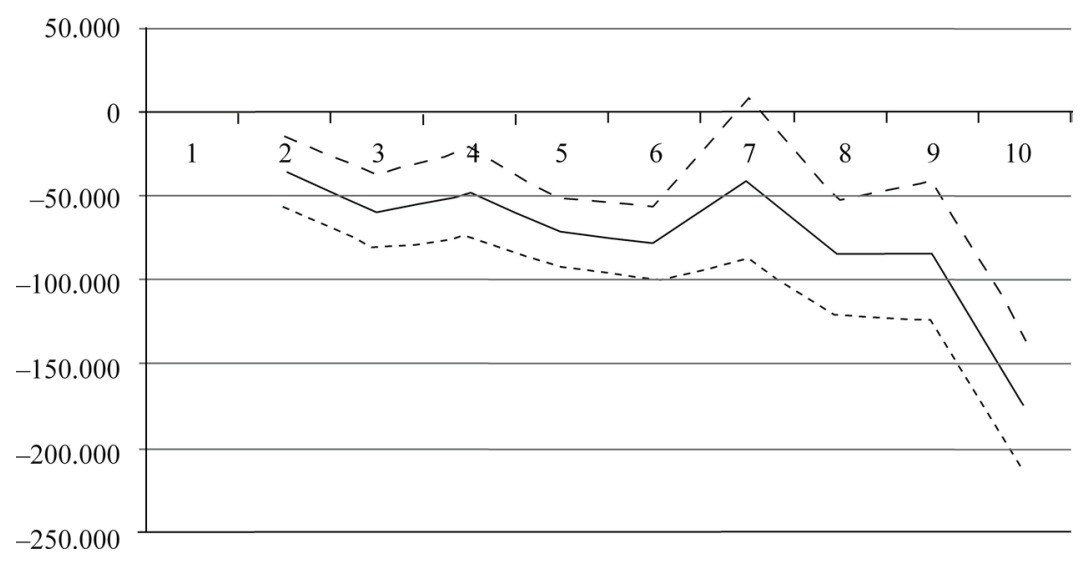

En conclusión, el estudio de las variaciones en el ingreso para las transiciones 1996-2001, 2001-2006 y 1996-2006 presenta los siguientes resultados: primero, para las tres transiciones estudiadas, la probabilidad de que los ingresos crezcan es mayor que la probabilidad de que los ingresos caigan; segundo, que aquellos individuos cuyos ingresos cayeron en la primera transición tienen una probabilidad significativamente mayor de que sus ingresos aumenten en la segunda; y tercero, que los individuos con mayores ingresos tienen una mayor probabilidad de sufrir una caída en sus ingresos que los individuos de menores ingresos. Estos resultados apoyan lo encontrado anteriormente: por un lado refutan la noción de que existen barreras que impiden la caída de los ingresos de los individuos de los deciles más ricos, y por el otro, muestran más bien que la movilidad observada en Chile es un fenómeno con fuertes componentes de regresión a la media, particularmente para los individuos de los deciles más extremos en la distribución del ingreso. El proceso de ingresos en Chile tiene alto grado de movilidad porque está dotado de un importante proceso de reversión a la media. O sea, como se vio en la primera sección, el proceso chileno tiene alto grado de independencia temporal (es lo que miden los índices estimados) y ahora esta sección muestra que también hay un importante 
grado de reversal. Esto, al parecer, se aleja de la noción de que la movilidad es algo indeseable que algunos, como se ha argumentado en el trabajo, quieren encontrar en dicho proceso.

\section{Conclusión}

Las secciones anteriores han presentado evidencia de que Chile exhibe altos niveles de movilidad intrageneracional del ingreso, y de que esta movilidad muestra que hay un componente importante de independencia temporal en la distribución del ingreso para Chile. Esto implica que, en el tiempo, se observan movimientos significativos a lo largo de la distribución del ingreso, lo que implica que la noción de que Chile es una sociedad económicamente rígida tiene poco asidero empírico.

Más aún, se ha mostrado que la movilidad observada para las transiciones 1996-2001, 2001-2006 y 1996-2006 puede ser replicada sin suponer que existen barreras al movimiento entre deciles. Esto es interesante, por cuanto se ha postulado que podrían existir barreras que entorpecen la movilidad desde y hacia el décimo decil. Este trabajo muestra que esto no es necesariamente verdadero, lo que vuelve a quitarle validez a la noción de que la sociedad chilena es económicamente rígida.

Finalmente, el estudio de los cambios en los ingresos de los individuos encuestados en la Encuesta Panel Casen muestra que la movilidad tiene un componente importante de regresión a la media, y que la probabilidad de que los ingresos aumenten en una transición es significativamente mayor si los ingresos cayeron en la transición anterior. Este resultado tiene una arista positiva y otra negativa: por un lado, implica que los individuos en los estratos más bajos de la sociedad tienen una alta probabilidad de surgir, lo que permitiría señalar que en muchos casos los episodios de caídas en los ingresos son transitorios; pero por otro, muestra que los individuos que han logrado acceder a los deciles más altos enfrentan una probabilidad importante de caer hacia rangos de ingreso menores. Lo interesante de este resultado es que implica que el componente de reversal de la movilidad intrageneracional del ingreso es significativo para Chile, por lo que se observaría una combinación de los dos atributos señalados como deseables por la literatura. 
Como conclusión, la combinación de los distintos argumentos presentados anteriormente apoya la noción de que Chile es una sociedad económicamente móvil. Este resultado es un aporte significativo para el estudio de la desigualdad en Chile, pues muestra que si bien la distribución del ingreso en nuestro país presenta un nivel alto de desigualdad, en el tiempo los individuos se mueven significativamente a lo largo de ésta.

\section{APÉNDICE}

Índices de movilidad intrageneracional del ingreso

El índice de Shorrocks calcula $S=\frac{n-\operatorname{tr}(P)}{n-1}$, donde n es el número de grupos en que se divide la distribución del ingreso (filas de la matriz de transición). Está acotado entre 0 y $\mathrm{n} /(\mathrm{n}-1)$, pues $\operatorname{tr}(\mathrm{P})=\mathrm{n}$ implica que todos los individuos se mantienen en el mismo decil, por lo que no hay movilidad y $\mathrm{S}=0$, mientras que $\operatorname{tr}(\mathrm{P})=0$ implica movilidad total $\mathrm{y}$ $\mathrm{S}=\mathrm{n} /(\mathrm{n}-1)$. En este caso, $\mathrm{n}=10$.

En tanto, el índice de Bartholomew calcula $\mathrm{B}=\sum_{\mathrm{i}=1}^{\mathrm{n}} \sum_{\mathrm{j}=1}^{\mathrm{n}} \mathrm{p}_{\mathrm{ij}}|\mathrm{i}-\mathrm{j}|$, y está acotado entre cero e infinito. Cuando no hay movilidad, todos los individuos están en la diagonal, por lo que $\mathrm{B}=0$, mientras que no es posible identificar una cota para el índice si todos los individuos están fuera de la diagonal.

Finalmente, el Immobility Ratio es simplemente la suma de la diagonal principal de la matriz de transición. A mayor valor de dicha suma, hay mayor rigidez intertemporal. Este índice está acotado entre cero y uno.

\section{REFERENCIAS}

Ayala, L., C. Navarro y M. Sastre (2002). “Cross-Country Income Mobility Comparisons Under Panel Attrition: The Relevance of Weighting Schemes". Working Papers 19-06, Instituto de Estudios Fiscales.

Bartholomew, D. J. (1973). Stochastic Models of Social Processes. London: Wiley, $2^{\text {da }}$ edición.

Castro, R. (2008). "Pobreza y Movilidad de Ingresos en Chile". Serie Informe Social $\mathrm{N}^{\circ} 111$, Libertad y Desarrollo. 
Chen, W-H. (2006). "Canadian Income Mobility in a Cross-National Perspective: Are We So Different?". Family and Labour Studies, Statistics Canada.

Contreras, D., R. Cooper, J. Herman y C. Neilson (2004). "Dinámica de la Pobreza y Movilidad Social”. Departamento de Economía de la Universidad de Chile, agosto 2004.

Contreras, D., M. Fuenzalida y J. Núñez (2006). "Persistencia Intergeneracional del Ingreso en Chile y el Rol de la Habilidad de los Hijos". Tesis de Magíster, Departamento de Economía, Universidad de Chile.

Corak, M. (2006). "Do Poor Children Become Poor Adults? Lessons from a Cross Country Comparison of Generational Earnings Mobility”. IZA Discussion Paper Series N 1993.

Denis, A., J. J. Prieto y J. R. Zubizarreta (2007). "Dinámica de la Pobreza en Chile: Evidencias en los Años 1996, 2001 y 2006”. Persona y Sociedad, Vol. 21, No 3 .

Fields, G y E. Ok (1996). "The Meaning and Measurement of Income Mobility". Journal of Economic Theory, 71, 349-377. - (2001). "The Measurement of Income Mobility: An Introduction to the Literature". En Jacques Silber (ed.), Inequality Measurement: From Theory to Practice. New York: Springer Science Business Media.

Gottschalk, P. y E. Spolaore (2002). "On the Evaluation of Economic Mobility”. The Review of Economic Studies, Vol. 69, N 1 (Jan. 2002), pp. 191-208.

Grawe (2006). "Lifecycle Bias in Estimates of Intergenerational Earnings Persistence". Labour Economics 13 (2006), 551-570.

Haider, S. y G. Solon (2006). "Life-Cycle Variation in the Association between Current and Lifetime Earnings". Working Paper 11943, National Bureau of Economic Research.

Hausman, Jerry (2001). "Mismeasured Variables in Econometric Analysis: Problems from the Right and Problems from the Left". Journal of Economic Perspectives Vol. 15(4) Fall, 57-67.

Hertz et al. (2007). "The Inheritance of Educational Inequality: International Comparisons and Fifty-Year Trends". The B. E. Journal of Economic Analysis and Policy, Vol.7, $\mathrm{N}^{\circ} 2$.

Mazumder, B. (2005). "Fortunate Sons: New Estimates of Intergenerational Mobility in the United States Using Social Security Earnings Data". The Review of Economics and Statistics, May 2005, 87 (2): 235-255.

Núñez, J. y L. Miranda (2006). "Recent Findings on Intergenerational Income and Educational Mobility in Chile". Working Paper $N^{\circ} 244$, Department of Economics, Universidad de Chile.

Núñez, J. y C. Risco (2004). "Movilidad Intergeneracional de Ingresos en un País en Desarrollo: El Caso de Chile". Working Paper $\mathrm{N}^{\circ} 210$, Department of Economics, Universidad de Chile.

OECD (2002). Education at a Glance: OECD Indicators 2002.

Paredes, R., J. J. Prieto y J. R. Zubizarreta (2006). "Attrition in Longitudinal Data and Income Mobility in Chile". Mimeo, Observatorio Social, Universidad Alberto Hurtado. Santiago de Chile, 2006. 
Regoli, A., C. Quintano y R. Castellano (2003). "Income Mobility in Italy". Hawaii International Conference on Statistics and Related Fields, June 5-8, 2003.

Sastre, M. y L. Ayala (2002). "Europe vs. the United States: Is There a Tradeoff between Mobility and Inequality?". Working Paper No. 2002-26, University of Essex, Institute for Social and Economic Research, U. K.: Colchester.

Sapelli, C. (2008). "Evolución de la Movilidad Intergeneracional de la Educación por Cohortes: Chile 1930-1981”. Presentado a la Sechi, septiembre 2007.

Solon, G. (1992). "Intergenerational Mobility in the United States". The American Economic Review, Vol. 82, N 3.

(1999). "Intergenerational Mobility in the Labor Market". En O. Ashenfelter y D. Card (eds.), Handbook of Labor Economics. Volume 3. (2002). "Cross-Country Differences in Intergenerational Earnings Mobility". The Journal of Economic Perspectives, Vol. 16, № 3.

Shorrocks, A. F. (1978a). "Income Inequality and Income Mobility", Journal of Economic Theory, 46, 566-578.

(1978b). "The Measurement of Mobility". Econometrica, 46, 10131024. 